\title{
Air, Helium and Water Leakage in Rubber 0-ring Seals with Application to Syringes
}

\author{
C. Huon ${ }^{1} \cdot$ A. Tiwari ${ }^{2,3} \cdot$ C. Rotella ${ }^{1} \cdot$ P. Mangiagalli ${ }^{1}$ B. N. J. Persson ${ }^{2,3}$
}

Received: 14 October 2021 / Accepted: 14 January 2022 / Published online: 23 February 2022

(c) The Author(s) 2022, corrected publication 2022

\begin{abstract}
We study the leakage of fluids (liquids or gases) in syringes with glass barrel, steel plunger and rubber O-ring stopper. The leakrate depends on the interfacial surface roughness and on the viscoelastic properties of the rubber. Random surface roughness is produced by sandblasting the rubber O-rings. We present a very simple theory for gas flow which takes into account both the diffusive and ballistic flow. The theory shows that the interfacial fluid flow (leakage) channels are so narrow that the gas flow is mainly ballistic (the so called Knudsen limit). We compare the leakrate obtained using air and helium. For barrels filled with water we observe no leakage even if leakage occurs for gases. We interpret this as resulting from capillary (Laplace pressure or surface energy) effects.
\end{abstract}

Keywords Syringes $\cdot$ Seals $\cdot$ Helium leakage $\cdot$ Ballistic gas flow $\cdot$ Interfacial separation $\cdot$ Viscoelasticity

\section{Introduction}

All solids have surface roughness which has a huge influence on a large number of physical phenomena such as adhesion, friction, contact mechanics and the leakage of seals [1-8]. Thus when two solids with nominally flat surfaces are squeezed into contact, unless the applied squeezing pressure is high enough, or the elastic modulus of at least one of the solids low enough, a non-contact region will occur at the interface. If the non-contact region percolate open flow channels exist, extending from one side of the nominal contact region to the other side. This will allow fluid to flow at the interface from a high fluid pressure region to a low pressure region.

For elastic solids with randomly rough surfaces the contact area percolate when the relative contact area $A / A_{0} \approx 0.42$ (see [9]), where $A_{0}$ is the nominal contact area and $A$ the area of real contact (projected on the $x y$-plane).

B. N. J. Persson

b.persson@fz-juelich.de

1 Sanofi, 13, Quai Jules Guesde-BP, 14-94403 Vitry-sur-Seine, Cedex, France

2 Peter Grünberg Institute (PGI-1), Forschungszentrum Jülich, 52425 Jülich, Germany

3 Multiscale Consulting, Wolfshovener str. 2, 52428 Jülich, Germany
When the contact area percolate there is no open (non-contact) channel at the interface extending across the nominal contact region, and no fluid can flow between the two sides of the nominal contact.

The discussion above is fundamental for the leakage of static seals. Here we are interested in rubber seals, e.g., rubber O-ring seals, or rubber stoppers for syringes. In the latter application, the contact between the ribs on the rubber stopper and the (glass or polymer) barrel must be so tight that no or negligible fluid can flow from inside the syringe to the outside. In addition, container closure integrity is very important so that no microorganism can penetrate from the outside to inside the syringe. Since the smallest microorganism (prion) may be only $\approx 10 \mathrm{~nm}$ in diameter (the smallest virus is a few times larger), it is clear that complete container closure integrity would imply that the most narrow junction (denoted critical junction) in the largest open (non-contact) interfacial channel should be at most $10 \mathrm{~nm}$. When this condition is satisfied, the fluid leakage is also negligible.

We have prepared model syringes to study the leakage of fluids (liquids or gases) in rubber seals. We have used glass barrels and steel plunger with two rubber O-rings as the stopper, see Fig. 1. The O-rings are sandblasted to give well-defined random surface roughness. The surface roughness of the O-rings is measured using a stylus instrument and the surface roughness power spectra is calculated. The He gas, air and water leakage rates are measured for O-rings 


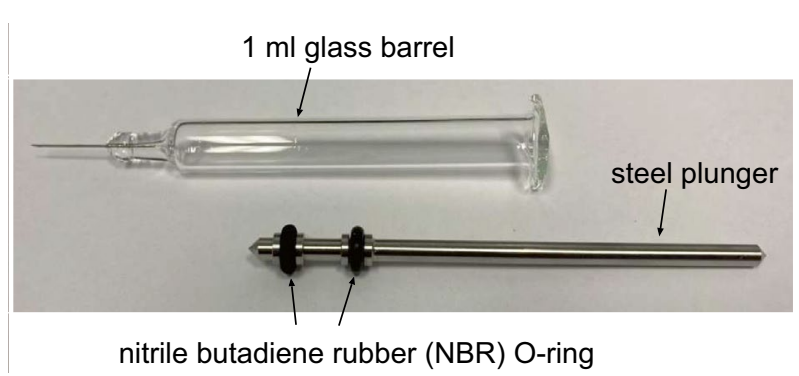

Fig. 1 A syringe used in the present experiments consisting of a $1 \mathrm{ml}$ glass barrel and a steel plunger with rubber O-rings. The O-rings are sandblasted to give well-defined random surface roughness

with different surface roughness. The leakrate depends on the interfacial surface roughness, but only weakly on the viscoelastic properties of the rubber. We show that the surface separation at the most narrow constrictions along the percolating interfacial fluid flow (leakage) channels are typically below $100 \mathrm{~nm}$. This is smaller than the (average) gas molecule mean free path (due to collisions between the gas molecules), which imply that the gas flow between the collisions with the solid walls is mainly ballistic rather than diffusive (the so called Knudsen limit). We present a very simple theory for the ballistic gas flow which is used in combination with the Persson contact mechanics theory to predict the helium (He), air and water leakrate. The calculated leakrates show good correlation with the measured leakrates.

\section{Leakrate Theory for Liquids}

In calculating the fluid (here air, He or water) leakrate we have used the effective medium approach combined with the Persson contact mechanics theory for the probability distribution of surface separations. The most important region for the sealing is a narrow strip at the center of the nominal contact region, where the contact pressure is highest (and the surface separation smallest), but the study presented below takes into account the full pressure profile $p(x)$.

The basic contact mechanics picture which can be used to estimate the leak-rate of seals is as follows: Consider first a seal where the nominal contact area is a square. The seal separate a high pressure fluid on one side from a low pressure fluid on the other side, with the pressure drop $\Delta p$. We consider the interface between the solids at increasing magnification $\zeta$. At low magnification we observe no surface roughness and it appears as if the contact is complete. Thus studying the interface only at this low magnification we would be tempted to conclude that the leak-rate vanishes. However, as we increase the magnification $\zeta$ we observe surface roughness and non-contact regions, so that the contact area $A(\zeta)$ is smaller than the nominal contact area $A_{0}=A(1)$. As we increase the magnification further, we observe shorter wavelength roughness, and $A(\zeta)$ decreases further. For randomly rough surfaces, as a function of increasing magnification, when $A(\zeta) / A_{0} \approx 0.42$ the non-contact area percolate [9], and the first open channel is observed, which allow fluid to flow from the high pressure side to the low pressure side. The percolating channel has a most narrow constriction over which most of the pressure drop $\Delta p$ occurs. In the simplest picture one assume that the whole pressure drop $\Delta p$ occurs over this critical constriction, and if it is approximated by a rectangular pore of height $u_{\mathrm{c}}$ much smaller than its width $w$ (as predicted by contact mechanics theory), the leakrate for a viscous fluid can be approximated by [10-16]

$\dot{Q}=\frac{u_{\mathrm{c}}^{3}}{12 \eta} \Delta p$

where $\eta$ is the fluid viscosity. The height $u_{\mathrm{c}}$ of the critical constriction can be obtained using the Persson contact mechanics theory (see Refs. [12, 17-20]). The result (1) is for a seal with a square nominal contact area. For a rectangular contact area with the length $L_{x}$ in the fluid flow direction and $L_{y}$ in the orthogonal direction, there will be an additional factor $L_{y} / L_{x}$ in (1). In a typical case the seal has a circular (radius $r_{0}$ ) cross section (like for rubber O-rings), and in this case $L_{y}=2 \pi r_{0}$ and typically $L_{y} / L_{x} \gg 1$ in which case the leak-rate will be much larger than given by the squareleak-rate formula (1). However, this geometrical correction factor is trivially accounted for. In deriving (1) it is assumed that the fluid pressure is negligible compared to the nominal contact pressure. If this is not the case one must include the deformation of the elastic solids by the fluid pressure distribution [21].

A more general and accurate derivation of the leakrate is based on the concept of fluid flow conductivity $\sigma_{\text {eff }}$. The fluid flow current

$J_{x}=-\sigma_{\text {eff }} \frac{d p_{\text {fluid }}}{d x}$

Since the leakrate $\dot{Q}=L_{y} J_{x}$ we get

$\frac{d p_{\text {fluid }}}{d x}=-\frac{\dot{Q}}{L_{y}} \frac{1}{\sigma_{\text {eff }}}$

Note that $\sigma_{\text {eff }}$ depends on the contact pressure $p_{\text {con }}(x)$ and hence on $x$. In the present case $p_{\text {fluid }} \ll p_{\text {cont }}$ and in this case $p_{\text {cont }} \approx p$, where $p(x)$ is the external applied squeezing pressure (or nominal contact pressure). Integrating (2) over $x$ gives

$\Delta p=\frac{\dot{Q}}{L_{y}} \int_{-\infty}^{\infty} d x \frac{1}{\sigma_{\text {eff }}(p(x))}$

where we have used that $\dot{Q}$ is independent of $x$ as a result of fluid volume conservation. For the Hertz contact pressure 
profile, where $p(x)=0$ for $x>a$ and $x<-a$, we get with $y=x / a$ :

$\Delta p=\dot{Q} \frac{L_{x}}{L_{y}} \int_{0}^{1} d y \frac{1}{\sigma_{\mathrm{eff}}(p(y))}$

where $L_{x}=2 a$ is the width of the contact region in the fluid flow direction and where

$p(y)=p_{0}\left(1-y^{2}\right)^{1 / 2}$

From (3) we get the fluid leakrate (volume per unit time)

$\dot{Q}=\frac{L_{y}}{L_{x}} \frac{\Delta p}{\int_{0}^{1} d y \sigma_{\text {eff }}^{-1}(p(y))}$

In the calculations presented below we have used (5) with the flow conductivity $\sigma_{\text {eff }}$ calculated using the Bruggemann effective medium theory ("corrected" so the contact area percolate for $A / A_{0}=0.42$; see Ref. [9]), and the Persson contact mechanics theory (see Refs. [11, 12] for the details). This theory takes into account all the fluid flow channels and not just the first percolating channel observed with increasing magnification as in the critical junction theory. The dependency of the leakrate on the fluid viscosity $\eta$ and the fluid pressure difference $\Delta p$ given by (1) is the same in the more accurate approach. Similar, the leak-rate is proportional to $L_{y} / L_{x}$ (where $L_{x}=2 a$ in the present case) in this more accurate approach.

\section{Leakage of Gases}

The leakage of gases is more complex than the leakage of liquids. The reason is that the separation between the solids in the critical junctions can be smaller than the mean free path $\lambda$ of the gas atoms (or molecules), due to collision between the gas atoms, which is of order $\approx 100 \mathrm{~nm}$ at room temperature and atmospheric pressures. When $u_{\mathrm{c}}<\lambda$ one need to take into account the ballistic motion of the gas atoms between the collisions with the solid walls. In particular, in experiments where the syringe is surrounded by vacuum the gas pressure may be much smaller (and the gas atom mean free path much bigger) close to the gas exit of the critical junction than that inside the syringe.

When ballistic effects are important one cannot use the fluid continuum equations to study the flow of the gas through the critical junction. This problem has been studied using the Boltzmann equation [22]. Here we will present two much simpler approaches to derive an approximate expression for the leakrate in the ballistic limit. We first briefly review some well-known facts about the kinetic theory of gases and the continuum (hydrodynamic) limit of gas flow.

\subsection{The Kinetic Theory of Gases}

We assume the ideal gas law

$p V=N k_{\mathrm{B}} T$,

or

$p=n k_{\mathrm{B}} T$,

where $n=N / V$ is the number of atoms per unit volume. The average gas atom velocity

$\bar{v}=\left(\frac{8 k_{\mathrm{B}} T}{\pi m}\right)^{1 / 2}$,

or

$k_{\mathrm{B}} T=\frac{\pi}{8} m \bar{v}^{2}$

where $m$ is the gas atomic mass.

Let $\sigma$ be the collision cross section of a gas atom. The atom mean free path

$\lambda=\frac{1}{\sqrt{2 n \sigma}}$.

The mean free path $\lambda$ is the distance an atom move (on the average) before it makes its first collision with another gas atom. The gas viscosity [23]

$\eta=\frac{1}{3} m n \bar{v} \lambda$

Using (6) this gives

$\eta=\frac{2}{3}\left(\frac{2}{\pi}\right)^{1 / 2} n \lambda\left(m k_{\mathrm{B}} T\right)^{1 / 2}$.

Note that since $\lambda \sim 1 / n$ the viscosity is independent of the gas density. Qualitatively it can be understood by considering the shearing of two parallel planes containing a gas inbetween. If the density of the gas is doubled, there are twice many molecules available to transport momentum from one plate to the other, but the mean free path of each molecule is also halved, so that it can transport this momentum only half as effectively.

In what follows we will assume that the temperature is constant. At room temperature for He the mean free path $\lambda \approx 174 \mathrm{~nm}$. Table 1 gives the viscosity and mean free path for several gases.

\subsection{Fluid Flow Through Rectangular Constriction}

Let us assume that the height $h$ of the rectangular constriction is much smaller than its width $w$ and length $L$ (see 
Table 1 Viscosity $\eta$, the gas atom mean free path $\lambda$ and the average gas atom (or molecule) speed $\bar{v}$ at room temperature and 1 atm pressure

\begin{tabular}{llll}
\hline gas & $\eta\left(10^{-5} \mathrm{Pas}\right)$ & $\lambda(\mathrm{nm})$ & $\bar{v}(\mathrm{~m} / \mathrm{s})$ \\
\hline $\mathrm{He}$ & 1.96 & 174 & 1245 \\
$\mathrm{~N}_{2}$ & 1.76 & 59 & 470 \\
$\mathrm{O}_{2}$ & 2.04 & 63 & 440 \\
$\mathrm{CO}_{2}$ & 1.47 & 39 & 375 \\
\hline
\end{tabular}

Fig. 2). Let $x$ be a coordinate axis along the constriction and assume the gas pressure $p=p_{\mathrm{b}}$ for $x=0$ and $p=p_{\mathrm{a}}$ for $x=L$. The atom number densities on the two sides are denoted $n_{\mathrm{b}}$ and $n_{\mathrm{a}}$.

We consider two limits, namely $\lambda \ll h$ and $\lambda \gg h$, where $\lambda$ is the mean free path. The first limit $\lambda \ll h$ can be studied using continuum fluid flow dynamics for a compressible fluid. In the other limit $\lambda \gg h$ ballistic flow occurs, where one can neglect the collisions between gas atoms and assume the gas atoms collide only with the solid walls (see Fig. 3). Note that ballistic refer only to the absence of collisions between the air molecules; the molecules may still scatter diffusely from the solid walls so the motion of gas molecules through the junction is always diffusive-like.

\subsubsection{Continuum Flow Limit $\lambda \ll h$}

For fluid flow between two parallel flat surfaces, if the separation $h \ll w$ and $h \ll L$, then the fluid velocity will vary much more rapidly in the normal direction $z$ then in the fluid flow direction $x$. In this case the Navier-Stokes equation reduces to

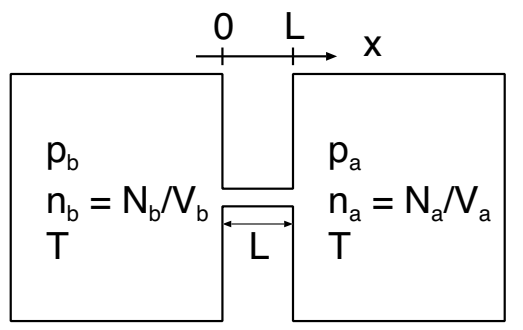

critical juction:

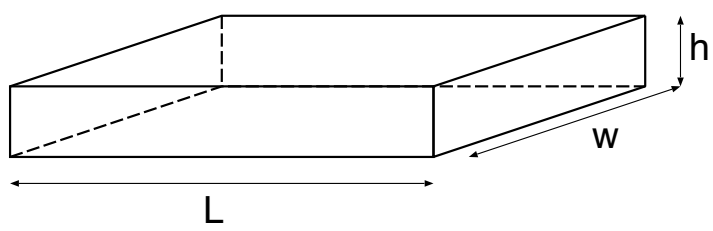

Fig. 2 A rectangular constriction between two volumes with gas at pressures $p_{\mathrm{a}}$ and $p_{\mathrm{b}}$ atom mean free path (in the gas) $=\lambda$

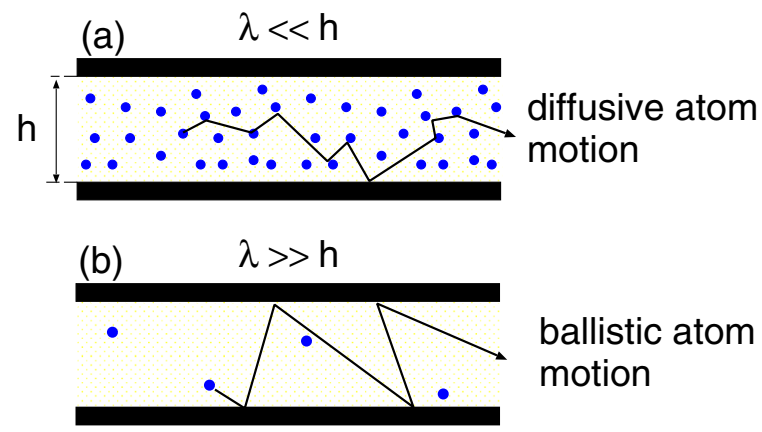

Fig. 3 Diffusive (a) and ballistic (b) motion of the gas atoms (e.g., $\mathrm{He}$ ) in the critical junction. In case a the gas mean free path $\lambda$ is much smaller than the gap width $h=u_{\mathrm{c}}$ and the gas molecules makes many collisions with other gas molecules before a collision with the solid walls. In the opposite limit, when $\lambda \gg u_{\mathrm{c}}$ the gas molecules makes many collisions with the solid walls before colliding with another gas molecule. In the first case a the gas can be treated as a (compressible) fluid, while a kinetic approach is needed in case $\mathbf{b}$

$\frac{\partial}{\partial z}\left(\eta \frac{\partial v_{x}}{\partial z}\right) \approx \frac{\partial p}{\partial x}$

and the fluid continuity equation

$\frac{\partial}{\partial x}\left(n v_{x}\right)=0$,

where $n$ is the gas number density (so $\rho=m n$ the gas mass density). Assuming an ideal gas the viscosity $\eta$ is independent of the gas density so that (11) takes the form

$\eta \frac{\partial^{2} v_{x}}{\partial z^{2}} \approx \frac{\partial p}{\partial x}$.

Since for an ideal gas $p=n k_{\mathrm{B}} T$ we can write (12) as

$\frac{\partial}{\partial x}\left(p v_{x}\right)=0$,

where we have assumed that the temperature is constant. We expect $p$ to depend mainly on $x$ and from (13) we get

$v_{x} \approx \frac{1}{2 \eta} z^{2} \frac{\partial p}{\partial x}+A z+B$

Since $v_{x}=0$ for $z=0$ and $z=h$ we get

$v_{x} \approx \frac{1}{2 \eta} z(z-h) \frac{\partial p}{\partial x}$

The flow current

$J_{x}=\int_{0}^{h} d z n v_{x} \approx-\frac{h^{3}}{12 \eta} n \frac{\partial p}{\partial x}$ 
must be independent of $x$, and using $p=n k_{\mathrm{B}} T$ this gives

$p \frac{\partial p}{\partial x}=C$

or

$p^{2}=2 C x+D$

Assuming $p=p_{\mathrm{b}}$ for $x=0$ and $p=p_{\mathrm{a}}$ for $x=L$ we get

$p^{2}=\left(p_{\mathrm{a}}^{2}-p_{\mathrm{b}}^{2}\right)(x / L)+p_{\mathrm{b}}^{2}$

Hence

$J_{x} \approx-\frac{h^{3}}{12 \eta} \frac{p}{k_{\mathrm{B}} T} \frac{\partial p}{\partial x}=\frac{h^{3}}{24 \eta L} \frac{p_{\mathrm{b}}^{2}-p_{\mathrm{a}}^{2}}{k_{\mathrm{B}} T}$

The number of atoms moving through the constriction per unit time equal $\dot{N}=w J_{x}$ or

$\dot{N}=\frac{h^{3} w}{24 \eta L} \frac{p_{\mathrm{b}}^{2}-p_{\mathrm{a}}^{2}}{k_{\mathrm{B}} T}$

In some leakage tests the syringe filled with gas (e.g., He) is put in vacuum. In this case $p_{\mathrm{a}}=0$ so that

$\dot{N}=\frac{h^{3} w}{24 \eta L} \frac{p_{\mathrm{b}}^{2}}{k_{\mathrm{B}} T}$

Using $p_{\mathrm{b}}=n_{\mathrm{b}} k_{\mathrm{B}} T$ and (6) and (9) this gives

$\dot{N}=\frac{\pi}{64} n_{\mathrm{b}} \bar{v} \frac{h^{3}}{\lambda_{\mathrm{b}}} \frac{w}{L}$

\subsubsection{Ballistic flow: qualitatively argument}

We consider the flow of gas atoms through the critical junction in the ballistic limit $\lambda \gg h$. Here we present a simple hand-waving argument: The flow of atoms into the junction from the high pressure side is $(1 / 4) n_{\mathrm{b}} \bar{v}$ times the area of the constriction given by $w h$. (The factor of $1 / 4$ is the result of the fact that only half of the atoms moves in the positive $x$-direction, and due to the fact that on the average they only move in the $x$-direction with the speed $\bar{v} / 2$.) Thus the number of atoms flowing into the constriction per unit time is $(1 / 4) n_{\mathrm{b}} \bar{v} w h$. However, the gas atoms will scatter from the walls in a random way (diffusive scattering) due to atomic surface roughness on the walls, and also due to the random thermal motion of the wall atoms. Thus some of the atoms which enter will get back-scattered and return to the high pressure volume $V_{\mathrm{b}}$ (see Fig. 4). It turns out only a fraction $2 h / L$ of the atoms will be able to penetrate the junction (see
Fig. 4 A gas atom which enter a constriction can be backscattered due to the atomic corrugation of the solid walls, or due to surface roughness or the thermal motion of the wall atoms
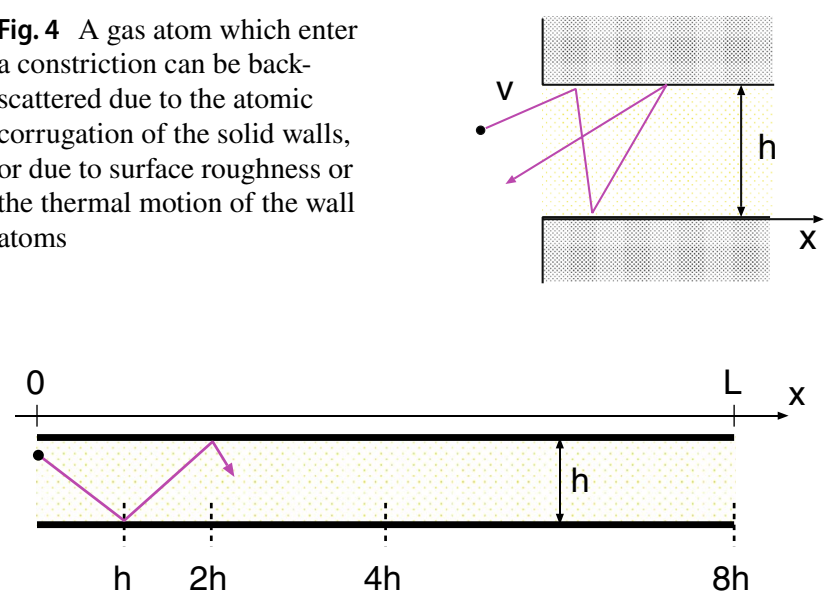

Fig. 5 An atom in the constriction collide with the walls in the channel at locations separated in the $x$-direction with on the average the distance $\approx h$. We assume diffusive scattering of the atom from the walls so when an atom is at $x=h$ (where it experience its first collision with a wall) it has equal probability to return to $x=0$ as to move to $x=2 h$. Hence the probability that it moves to $x=2 h$ is $1 / 2$. When it is at $x=2 h$ it has equal probability to return to $x=0$ as to move to $x=4 h$. Hence the probability to move to $x=4 h=2^{2} h$ will be $(1 / 2) \times(1 / 2)=(1 / 2)^{2}$. In the same way, the probability to move to $x=2^{n} h$ will be $(1 / 2)^{n}$. If it arrives to $x=L$ it is transferred from the high pressure side to the low pressure side. Thus the probability that an atom which enter the constriction at $x=0$ will exit it at $x=L$ will be $P=(1 / 2)^{n}$ where $2^{n} h=L$. Hence $P \approx h / L$

Fig. 5). Thus the number of atoms per unit time moving through the constriction will be

$\dot{N}=\frac{1}{2} n_{\mathrm{b}} \bar{v} h^{2} \frac{w}{L}$

\subsubsection{Ballistic Flow: Diffusion Equation}

We now present a more accurate derivation of the result (16). When $\lambda \gg h$ the leakage through a critical junction can be determined by a simple kinetic approach. We consider a junction with the height $h$, the width $w$ and the length (in the leakage flow direction) $L$. The average collision time for an atom in the gap with the walls is $\tau$, and we expect $\bar{v} \tau \approx h$, where $\bar{v}$ is the average atom velocity. Due to surface roughness, when an atom hit the wall it is reflected in a nearly random direction so sometimes in the leakage direction and sometimes in the opposite direction. The atom moves on the average a distance $\Delta x \approx h$ along or opposite to the leakage direction between each collisions with the wall. Let us use a discretized (in time and space) model and consider the probability $P(x, t)$ to find the particle at $x$ at time $t$. If the particle is at $x$ at time $t+\tau$ the particle must have been at either $x-\Delta x$ (with probability $1 / 2$ ) or at $x+\Delta x$ (with probability $1 / 2$ ) at time $t$. Thus we get 
$P(x, t+\tau)=\frac{1}{2} P(x-\Delta x, t)+\frac{1}{2} P(x+\Delta x, t)$

If we expand the probability to linear order in $\tau$ and quadratic order in $\Delta x$ we get a diffusion equation for $P(x, t)$ :

$\frac{\partial P}{\partial t}=\frac{\Delta x^{2}}{2 \tau} \frac{\partial^{2} P}{\partial x^{2}}$

Since the gas atoms does not interact with each other in the junction, we can also consider $P(x, t)$ as the concentration of atoms in the gap. If we denote this by $c(x, t)$ and if we use $\Delta x \approx h$ and $\bar{v} \tau \approx h$ we get

$\frac{\partial c}{\partial t}-D \frac{\partial^{2} c}{\partial x^{2}}=0$

where the diffusivity $D=\bar{v} h / 2$. The gas flow current $J$ satisfies the continuity equation

$\frac{\partial c}{\partial t}+\frac{\partial J}{\partial x}=0$

Comparing (17) and (18) gives

$J=-D \frac{\partial c}{\partial x}$

Here we are interested in a stationary state so that $c(x, t)$ is time independent. From (17) we get

$\frac{\partial^{2} c}{\partial x^{2}}=0$

i.e., $c(x)$ is a linear function of $x$. Assuming $c=n_{\mathrm{b}}$ for $x=0$, where $n_{\mathrm{b}}$ is the number of atoms per unit volume of the gas on the inlet side, and assuming that $c$ vanish (vacuum) on the exit side $x=L$, we get from (20):

$c(x)=n_{\mathrm{b}}\left(1-\frac{x}{L}\right)$

Using (19) this gives the current $J=D n_{\mathrm{b}} / L$. The gas leakage $\dot{N}$ is determined by the product between the current $J$ and the junction cross section area $w h$ so that $\dot{N}=J w h=D n_{\mathrm{b}} h w / L$. Using $D=\bar{v} h / 2$ this gives

$\dot{N}=\frac{1}{2} n_{\mathrm{b}} \bar{v} h^{2} \frac{w}{L}$,

which agree with (16).

In the derivation of (21) we have assumed a rectangular junction and that the molecules scatter randomly from the solid walls. Real junctions are not perfectly rectangular and the gas atoms may not scatter at random directions from the solid walls. Still, the theory we use gives good agreement with experiments which indicate that the idealized treatment is close to reality. We also note that the more accurate treatement based on the Boltzmann equation when compared to experiment shows that gas atoms typically scatter diffusively from solid walls with atomic roughness. Thus the study in Ref. [24, 25] showed best agreement with experiments when the tangential momentum accommodation coefficient $\alpha$ is between 0.8 and 1 , where $\alpha=1$ correspond to diffusive scattering and $\alpha=0$ to specular scattering.

\subsubsection{Interpolation Formula and Comparison with Experiments}

We can interpolate between the limits (15) and (21) using

$\dot{N}=\frac{\pi}{64} \frac{w}{L} h^{3} n_{\mathrm{b}} \bar{v}\left(\frac{1}{\lambda_{\mathrm{b}}}+\frac{\xi}{h}\right)$

where $\xi=32 / \pi$. We can interpret the factor

$\frac{1}{\lambda_{\mathrm{eff}}}=\frac{1}{\lambda_{\mathrm{b}}}+\frac{\xi}{h}$

as defining an effective mean free path.

The formula (22) gives leakage rates in close agreement with experiments and with the Boltzmann theory predictions assuming diffusive atom-wall scattering. Thus in Ref. [24, 25] experimental results was presented for rectangular channels with $w / h \approx 3$ but $L \gg w$. Our calculation is for $w / h \gg 1$ but theories have been developed for finite $w / h$ ratio [24], and taking this into account the experimental results presented in Ref. [25] is in good agreement with our theory prediction.

\subsubsection{Application to Syringe He-Gas Leakage Testing}

Helium is a very inert gas and it will not react with materials but it may inter-diffuse in some materials like polymers or rubber compounds. In most helium leak testing applications, one uses a mass spectrometer tuned to detect helium. High vacuum testing allows leak test down as low as $\dot{V} \approx 10^{-12} \mathrm{~cm}^{3} /$ s of He of atmospheric pressure.

Suppose we put a syringe (with closed needle) filled with He gas (volume $V_{\mathrm{b}}$ at pressure $p_{\mathrm{b}}$ ) in a closed volume $V_{\mathrm{a}}$ at pressure $p_{\mathrm{a}}=0$ (vacuum). Due to He gas leakage the pressure outside of the syringe will slowly increase. Since $p V=N k_{\mathrm{B}} T$ we get $\dot{p}_{\mathrm{b}} V_{\mathrm{b}}=\dot{N}_{\mathrm{b}} k_{\mathrm{B}} T$ and $\dot{p}_{\mathrm{a}} V_{\mathrm{a}}=\dot{N}_{\mathrm{a}} k_{\mathrm{B}} T$, where we have assumed that the temperature is constant. Since the gas atoms leaving the syringe enters the volume $V_{\mathrm{a}}$ we must have (conservation of atoms) $\dot{N}_{\mathrm{a}}=-\dot{N}_{\mathrm{b}}$ so that:

$\dot{p}_{\mathrm{a}} V_{\mathrm{a}}=-\dot{p}_{\mathrm{b}} V_{\mathrm{b}}=\dot{N}_{\mathrm{a}} k_{\mathrm{B}} T$

To apply this to syringes we use (22) with $w=L$ (square shaped critical junction), $h=u_{\mathrm{c}}$ and with an additional factor $L_{y} / L_{x} \approx 100$ due to the rectangular nature of the nominal rib-glass contact region. Thus the gas atom leakrate 
$\dot{N} \approx \frac{\pi}{64} u_{\mathrm{c}}^{3} n_{\mathrm{b}} \bar{v} \frac{L_{\mathrm{y}}}{L_{\mathrm{x}}}\left(\frac{1}{\lambda_{\mathrm{b}}}+\frac{\xi}{u_{\mathrm{c}}}\right)$

and (24) takes the form

$\dot{p}_{\mathrm{a}} V_{\mathrm{a}} \approx \frac{\pi}{64} u_{\mathrm{c}}^{3} n_{\mathrm{b}} \bar{v} \frac{L_{\mathrm{y}}}{L_{\mathrm{x}}}\left(\frac{1}{\lambda_{\mathrm{b}}}+\frac{\xi}{u_{\mathrm{c}}}\right) k_{\mathrm{B}} T$

Using that $p_{\mathrm{b}}=n_{\mathrm{b}} k_{\mathrm{B}} T$ we can also write (26) as

$\dot{p}_{\mathrm{a}} V_{\mathrm{a}} \approx \frac{\pi}{64} u_{\mathrm{c}}^{3} p_{\mathrm{b}} \bar{v} \frac{L_{\mathrm{y}}}{L_{\mathrm{x}}}\left(\frac{1}{\lambda_{\mathrm{b}}}+\frac{\xi}{u_{\mathrm{c}}}\right)$

In many syringe applications $\xi / u_{\mathrm{c}} \gg 1 / \lambda_{\mathrm{b}}$ and in this case (27) reduces to

$\dot{p}_{\mathrm{a}} V_{\mathrm{a}} \approx \frac{1}{2} u_{\mathrm{c}}^{2} p_{\mathrm{b}} \bar{v} \frac{L_{\mathrm{y}}}{L_{\mathrm{x}}}$

The quantity $\dot{p}_{\mathrm{a}} V_{\mathrm{a}}=\dot{N}_{\mathrm{a}} k_{\mathrm{B}} T$, with units $\mathrm{Pa} \times \mathrm{m}^{3} / \mathrm{s}$ or mbar $\times 1 / \mathrm{s}$, is often measured in He leakage experiments [26-28]. Sometimes the leakrate is given as volume of gas at the pressure $p_{\mathrm{b}}$ passing through the constriction per unit time. This is obtained from $\dot{N}$ using $V=N k_{\mathrm{B}} T / p_{\mathrm{b}}$ so that $\dot{V}=\dot{N} k_{\mathrm{B}} T / p_{\mathrm{b}}$, where we have assumed that $p_{\mathrm{b}}$ is constant. Often $\dot{V}$ is given as $\mathrm{cm}^{3} / \mathrm{s}$. Using (25) and $p_{\mathrm{b}}=n_{\mathrm{b}} k_{\mathrm{B}} T$ we get

$\dot{V} \approx \frac{\pi}{64} u_{\mathrm{c}}^{3} \bar{v} \frac{L_{\mathrm{y}}}{L_{\mathrm{x}}}\left(\frac{1}{\lambda_{\mathrm{b}}}+\frac{\xi}{u_{\mathrm{c}}}\right)$

If $\xi / u_{\mathrm{c}} \gg 1 / \lambda_{\mathrm{b}}(29)$ reduces to

$\dot{V} \approx \frac{1}{2} u_{\mathrm{c}}^{2} \bar{v} \frac{L_{\mathrm{y}}}{L_{\mathrm{x}}}$

We have observed (see also Ref. [27]) that syringes gives typical leakrates $\dot{p}_{\mathrm{a}} V_{\mathrm{a}}=(1-100) \times 10^{-8} \mathrm{mbar} \times 1 / \mathrm{s}$. If we assume $p_{\mathrm{b}}=0.1 \mathrm{MPa}$ (atmospheric pressure) and $p_{\mathrm{a}} \approx 0$ (vacuum) and $L_{y} / L_{x}=100$ we get from (28) that $u_{\mathrm{c}} \approx 4 \mathrm{~nm}$ if $\dot{p}_{\mathrm{a}} V_{\mathrm{a}}=10^{-6} \mathrm{mbar} \times \mathrm{l} / \mathrm{s}, u_{\mathrm{c}} \approx 1 \mathrm{~nm}$ if $\dot{p}_{\mathrm{a}} V_{\mathrm{a}}=10^{-7} \mathrm{mbar} \times 1 / \mathrm{s}$.

He atoms (and air molecules) can be absorbed by the rubber matrix and diffuse through the rubber stopper, and this too will contribute to the observed leakage. Let us estimate the leakage rate due to He-diffusion through the rubber. We consider a rib on the stopper which we treat as a rectangular region of height $d_{0}$ and length (in the gas diffusion direction which we take as the $x$-direction) $d_{1}$ and width $L_{y}=2 \pi R$. Hence the cross section area $A=d_{0} L_{y}$. We assume a steady state (see below) where the concentration of $\mathrm{He}$ atoms varies linearly between the inner surface of the rubber stopper and the outer surface. The He diffusion current
$J=-D_{\mathrm{g}} \frac{d c}{d x}=D_{\mathrm{g}} \frac{c_{\mathrm{b}}-c_{\mathrm{a}}}{d_{1}}$

where the gas diffusion coefficient $D_{\mathrm{g}}$ has the unit $\mathrm{m}^{2} / \mathrm{s}$. We assume that the concentration of $\mathrm{He}$ atoms at the inner and outer surfaces are proportional to the gas pressures inside and outside the syringe. In this case $c_{\mathrm{b}}=S p_{\mathrm{b}}$ (where $S$ is the solubility) and $c_{\mathrm{a}}=0$ since we assume $p_{\mathrm{a}}=0$ (vacuum). Thus we get

$\dot{N}=J A=D_{\mathrm{g}} S \frac{A}{d_{1}} p_{\mathrm{b}}$

The quantity $\kappa=D_{\mathrm{g}} S k_{\mathrm{B}} T$ (with units $\mathrm{m}^{2} / \mathrm{s}$ ) is denoted the gas permeability. Using $\dot{p}_{\mathrm{a}} V_{\mathrm{a}}=\dot{N} k_{\mathrm{B}} T$ we get

$\dot{p}_{\mathrm{a}} V_{\mathrm{a}}=\kappa \frac{A}{d_{1}} p_{\mathrm{b}}$

Depending on the type of rubber, for $\mathrm{He}$ $\kappa=(3-30) \times 10^{-12} \mathrm{~m}^{2} / \mathrm{s}$ (see Ref. [29]), while for $\mathrm{N}_{2}$ the gas permeability may be $\sim 10$ times smaller. We consider the He gas leakage through a rib treated as a rectangular region with height $d_{0}$, length (in the leakage direction) $d_{1}$ and width $L_{y}=2 \pi R$ we get $A / d=2 \pi R d_{0} / d_{1}$. Typically, $d_{0} \approx d_{1}$ so we get

$\dot{p}_{\mathrm{a}} V_{\mathrm{a}} \approx \kappa 2 \pi R p_{\mathrm{b}}$

Using $R=0.5 \mathrm{~cm}$ this gives

$\dot{p}_{\mathrm{a}} V_{\mathrm{a}} \approx 10^{-7}-10^{-6} \mathrm{mbar} \cdot \mathrm{l} / \mathrm{s}$

This is similar to the measured leakage rates for syringes. Thus, in many cases the leakage will be strongly influenced by diffusion of He through the rubber matrix.

Finally, let us determine how long time it takes for diffusion through the rubber to reach the steady state. Assume that the $\mathrm{He}$ gas in introduced in the syringe at time $t=0$. From the diffusion equation we know (from dimensional arguments) that it will take the time $t_{0} \approx d_{1}^{2} / D_{\mathrm{g}}$ to reach the steady state. For He in rubber $D_{\mathrm{g}} \approx 10^{-10} \mathrm{~m}^{2} / \mathrm{s}$ and with $d_{1} \approx 1 \mathrm{~mm}$ this gives $t_{0} \approx 10^{4} \mathrm{~s}$. A more accurate analysis gives a factor $\sim 6$ shorter time so we conclude it typically take $\approx 1 \mathrm{~h}$ to reach the steady state. In most leakage studies the observation time might be much smaller than this, and in these cases a smaller (and time dependent) leakrate will be observed. We note for $t<0.1 d_{1}^{2} / D_{\mathrm{g}}$ theory predict negligible leakage resulting from $\mathrm{He}$ diffusion through the rubber. On the other hand the gas leakage through the open (non-contact) channels at the rubber-barrel interface occurs immediately after filling the syringe with gas. It is clear that the time dependency of the leakrate may contain very important information about the origin of the leakage.

We note that leakage of air through most types of rubber is slower than for He. One well studied case is the 


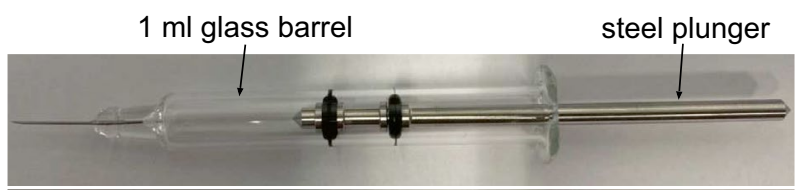

Fig. 6 Steel plunger with two rubber NBR O-rings in a glass barrel

diffusion of different gas molecules in ethylene-propylenediene (EPDM) elastomer [30]. The diffusivity at room temperature for $\mathrm{He}$ and $\mathrm{N}_{2}$ in EPDM are $D_{\mathrm{g}}=1.7 \times 10^{-9}$ and $5.5 \times 10^{-11} \mathrm{~m}^{2} / \mathrm{s}$. The higher diffusivity for He reflects its smaller effective size (effective van der Waals diameter of $\mathrm{He}$ and $\mathrm{N}_{2}$ are $2.8 \mathrm{~nm}$ and $3.8 \mathrm{~nm}$, respectively). However, the solubility for He in EPDM is $\approx 5$ times lower than for $\mathrm{N}_{2}$ so the He permeability $\kappa=D_{\mathrm{g}} S k_{\mathrm{B}} T$ is only $\sim 6$ times bigger than for $\mathrm{N}_{2}$.

\section{Leakage experiments with syringes with rubber O-ring stopper}

Most syringes exhibit gas leakage which is too small to be accurately studied and which may result from diffusion of gas through the rubber, or of some other origin. To obtain larger leakage rates experiments have been performed where a thin metal wire is located between the rubber stopper and the barrel [31]. Here we instead use rubber stoppers which are sandblasted to produce random roughness. We used a steel plunger with two rubber O-rings and a $1 \mathrm{ml}$ glass barrel (see Fig. 6). We use O-rings with the inner diameter $D=3 \mathrm{~mm}$, and the rubber cross section diameter $2 R=2 \mathrm{~mm}$. Hence the O-ring outer diameter $d=D+4 R=7 \mathrm{~mm}$. However, the sandblasted O-rings have smaller outer diameter. We have sandblasted O-rings made from 6 different types of rubber (exhibiting very different wear rates [32]). We have measured the surface topography of the sandblasted surfaces. We have also measured the viscoelastic modulus of the O-rings rubber compounds. Here we show the results obtained for O-rings made from nitrile butadiene rubber (NBR70).

\subsection{Rubber Viscoelastic Modulus}

We use rubber O-rings made from nitrile butadiene rubber (NBR70) with the glass transition temperature $T=-34.3^{\circ} \mathrm{C}$. The low strain and low frequency $\left(\omega \approx 10^{-3} \mathrm{~s}^{-1}\right.$, corresponding to the waiting time $t \approx 1 \mathrm{~h}$ ) elastic modulus is about $25 \mathrm{MPa}$ but due to strain softening for a more relevant strain (of order $50 \%$ ) the effective modulus is $\approx 7 \mathrm{MPa}$ (see Fig. 7). As will be shown below, in the analysis presented the actual value of the elastic modulus is irrelevant as long as the strain in the (macroscopic) nominal contact area is very

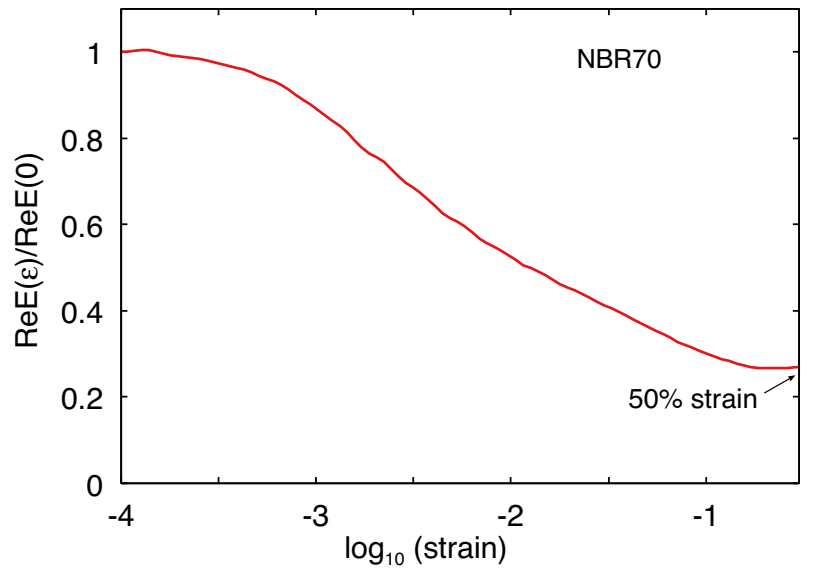

Fig. 7 Strain softening. The ratio between the real part of the viscoelastic modulus for zero strain and the strain $\epsilon$, as a function of the logarithm of the strain. The results is for NBR70 at $T=20^{\circ} \mathrm{C}$ and for the frequency $f=1 \mathrm{~Hz}$

Table 2 The root-mean-square (rms) roughness amplitude $h_{\text {rms }}$ (in $\mu \mathrm{m}$ ) and the rms 2D-slope for the studied O-rings ordered after increasing $h_{\mathrm{rms}}$

\begin{tabular}{lll}
\hline O-ring & $h_{\text {rms }}(\mu \mathrm{m})$ & rms-slope \\
\hline O0 & 2.21 & 0.09 \\
O3 & 3.02 & 0.33 \\
O9a & 3.45 & 0.30 \\
O4 & 3.46 & 0.34 \\
O5 & 3.50 & 0.33 \\
O1 & 3.69 & 0.36 \\
O8a & 3.81 & 0.36 \\
O2 & 4.46 & 0.48 \\
O6a & 5.02 & 0.48 \\
O3a & 5.80 & 0.51 \\
O10a & 6.34 & 0.87 \\
\hline
\end{tabular}

The O-ring O0 is not sandblasted

similar to the typical strain involved in the asperity contact regions (Table 2).

\subsection{Sandblasting and Rubber Surface Roughness}

We had sandblasted two sets of 10 NBR70 rubber O-rings which we denote by $\mathrm{O} 1-\mathrm{O} 10$ and $\mathrm{O} 1 \mathrm{a}-\mathrm{O} 10 \mathrm{a}$ (the two sets of O-rings was sandblasted at different days). The sandblasting was performed by putting a rubber O-ring on a steel rod which is attached to an electric drill which rotates the steel rod at about 1 rotation per second, while it was exposed to a beam of spherical glass particles with a diameter of order a few micrometer. The beam of particles was centered on the rubber O-ring and the sandblasting air pressure was about $10 \mathrm{bar}$, and the sandblasting time was varied between 10 
minutes and 30 minutes in order to obtain O-rings with different surface roughness and effective radius.

We have measured the surface roughness profile using a stylus instrument [Mitutoyo Portable Surface Roughness Measurement Surftest SJ-410 with a diamond tip with the radius of curvature $r_{0}=1 \mu \mathrm{m}$, and with the tipsubstrate repulsive force $F_{\mathrm{N}}=0.75 \mathrm{mN}$ and the tip speed $v=50 \mu \mathrm{m} / \mathrm{s}]$, and calculated the surface roughness power spectrum as described in detail elsewhere [6]. Figure 8 shows the 1D surface roughness power spectra of the sandblasted O-rings O1-O5, and for a not sandblasted O-ring. Figure 9 shows the 2D surface roughness power spectra obtained from 1D line scans from the sandblasted rubber O-rings O3a, O6a, O8a, O9a, O10a. In each case the power spectra was obtained by averaging over 7 line scans, each $2 \mathrm{~mm}$ long in the direction of the largest O-ring curvature radius.

Figure 10 shows the 2D surface roughness power spectra obtained from 1D line scans from the sandblasted rubber O-ring O3a. The thick red line is the average power spectrum, obtained by average the power spectra obtained from 7 line scans. The thin lines gives the power spectra of each individual line scan (each $2 \mathrm{~mm}$ long).

\subsection{Optical Pictures of the Nominal Rubber-Barrel Contact Region}

The sandblasting results in a decrease in the effective O-ring radius and hence a change in the width of the Hertz-like contact between the O-ring on the steel plunger and the glass barrel. This is illustrated in Fig. 11c for a not sandblasted rubber O-ring, and in Fig. 11b for a strongly sandblasted O-ring. The width of the nominal contact area for the sandblasted O-ring is much smaller than for the not sandblasted O-ring ( $0.31 \mathrm{~mm}$ and $1.37 \mathrm{~mm}$, respectively). In the

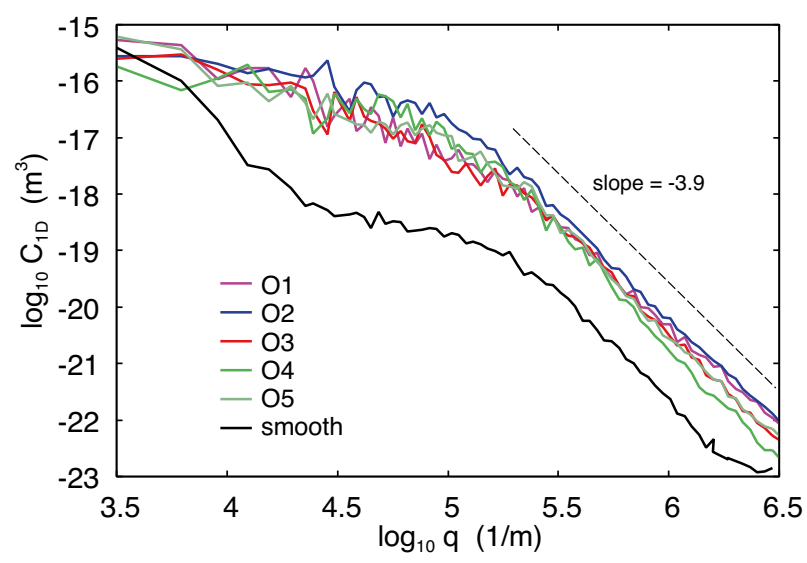

Fig. 8 The 1D surface roughness power spectra of the sandblasted O-rings $\mathrm{O} 1-\mathrm{O} 5$, and for a not sandblasted (smooth) O-ring

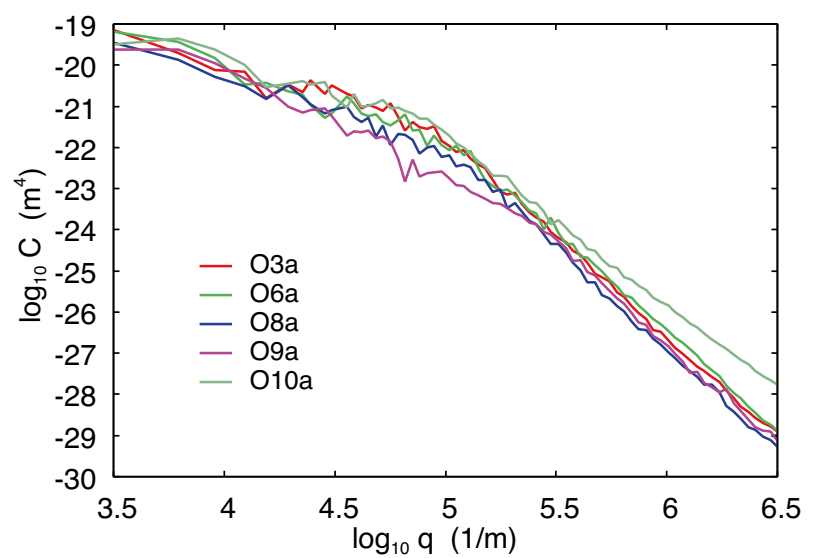

Fig. 9 The 2D surface roughness power spectra obtained from 1D line scans from the sandblasted rubber O-rings O3a, O6a, O8a, O9a, O10a. In each case the power spectra was averaged over 7 line scans each $2 \mathrm{~mm}$ long in the direction of the largest O-ring curvature radius

experiments presented below the outer (not sandblasted) O-ring was cut with a scalpel in such a way that a thin open channel occurred in the rubber-glass nominal contact region. Hence, the whole pressure drop in the leakage experiments occurs over the inner sandblasted O-ring.

We have measured the width of the rubber-glass contact region for all the O-rings used in the leakage studies. The measurements was performed both in Jülich and at Sanofi using two different optical microscope. Figure 12 shows the results obtained in Jülich for rubber O-rings O8a and O10a and for a not sandblasted O-ring. In Table 3 we show the width $w$ of the contact region for all the studied O-rings as measured in Jülich. Using the Hertz theory for cylinder

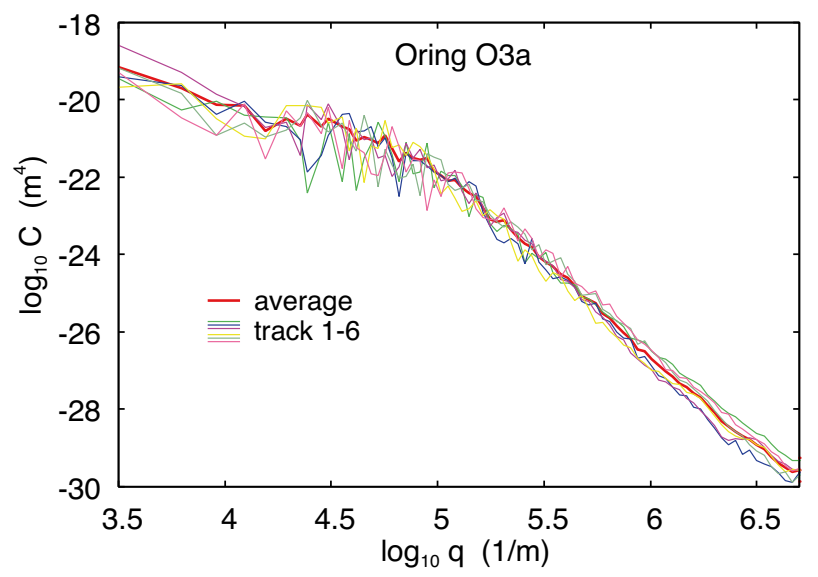

Fig. 10 The 2D surface roughness power spectra obtained from 1D line scans from the sandblasted rubber O-ring O3a. The thick red line is the power spectrum obtained by averaging the power spectra obtained from 7 line scans each $2 \mathrm{~mm}$ long in the direction of the largest O-ring curvature radius. Then thin lines gives the power spectra of each individual line scan 


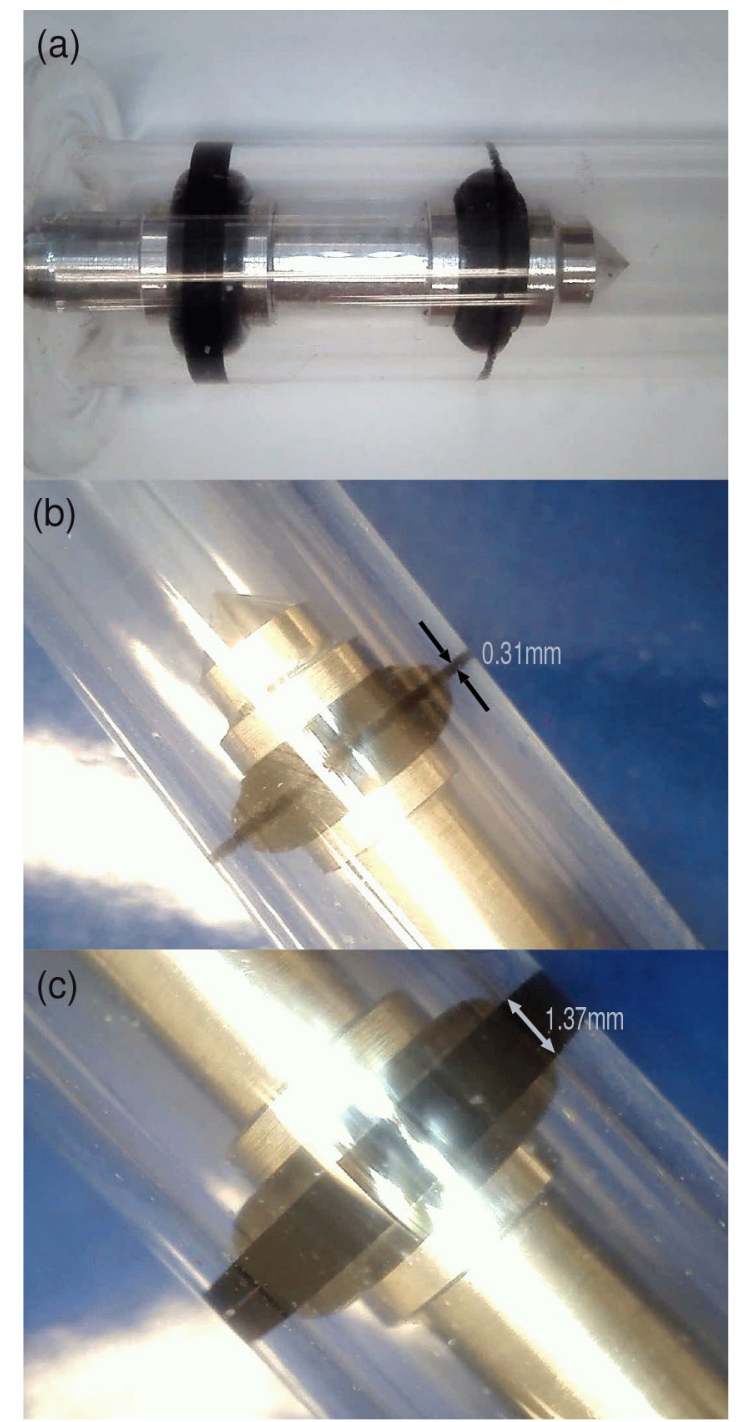

Fig. 11 Optical pictures of two NBR70 rubber O-rings on a steel stopper inserted in a glass barrel. The front O-ring is sandblasted which reduces the outer radius of the O-ring and results in a reduced width of the rubber-glass nominal contact region. a Shows both O-rings in the glass barrel and $\mathbf{b}$ and $\mathbf{c}$ the contact with the sandblasted and not sandblasted O-ring, respectively

contact, from the width of the contact region we can calculate the penetration $\delta=w^{2} /(4 R)$, the loading force per unit length $F / L=(\pi / 4) E^{*} \delta$, the maximum contact pressure $p_{0}=\left(E^{*} F / \pi R L\right)^{1 / 2}$ and the strain $2 \delta / w$. In the calculations we have used $R=1 \mathrm{~mm}$ and $E_{1}=E /\left(1-v^{2}\right)$ with $E=7 \mathrm{MPa}$ and $v=0.5$. In calculation of the leakage rates presented below we have used (5) and assumed that the pressure distribution $p(x)$ is of the Hertz type (4) with the width $L_{x}=w$ and the maximum pressure $p_{0}$ given in Table 3 .

We have used the same radius of curvature $R$ for the sandblasted O-rings as for the not sandblasted O-ring. For the sandblasted O-rings we expect $R$ to be somewhat smaller than $1 \mathrm{~mm}$, but the cross section of these O-rings
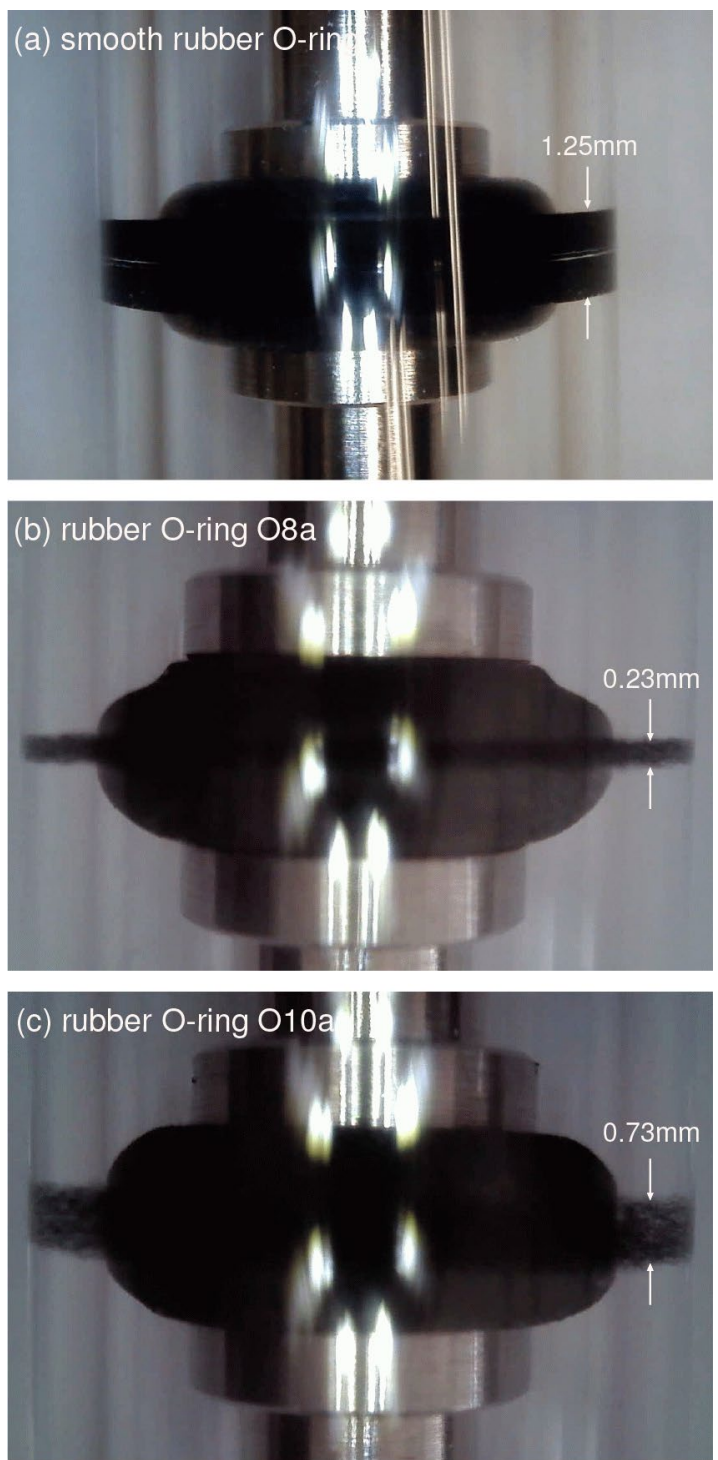

Fig. 12 Optical picture of a a not sandblasted (smooth) O-ring and of sandblasted O-ring O8a (b) and O10a (c) in the glass barrel. The width of the contact region are $w \approx 1.25,0.23$ and $0.73 \mathrm{~mm}$, respectively

is not circular so $R$ cannot be accurately deduced just from the change in the O-ring cross section. The most important contact mechanics quantity for the leakrate is the maximum contact pressure which is proportional to $E^{*} w / R$ for the Hertz contact between a cylinder and a flat. In this equation the width $w$ of the contact changes a lot as a result of the sandblasting and range from $0.75 \mathrm{~mm}$ for O-ring $\mathrm{O} 5$ to $0.26 \mathrm{~mm}$ for O-ring $\mathrm{O} 4$ (see Table 3). However, the rather small change in the (calculated) penetration from $0.14 \mathrm{~mm}$ to $0.02 \mathrm{~mm}$ correspond to a reduction in the radius $R$ by about $0.12 \mathrm{~mm}$. Hence while $w$ may change with a factor of $\sim 3$ the radius may change with $\sim 10-15 \%$ and the ratio $w / R$ varies mainly due the large variation in $w$. 
Table 3 The measured contact width $w$ and the calculated penetration $\delta$, normal force per unit length $F / L$, maximum contact pressure $p_{0}$, and the strain in the contact region for the studied O-rings

\begin{tabular}{llllll}
\hline O-ring & $w(\mathrm{~mm})$ & $\delta(\mathrm{m} \mathrm{m})$ & $F / L(\mathrm{~N} / \mathrm{m})$ & $p_{0}(\mathrm{MPa})$ & Strain $(\%)$ \\
\hline O0 & 1.25 & 0.391 & 2863 & 2.92 & 63 \\
O5 & 0.75 & 0.141 & 1031 & 1.75 & 38 \\
O6a & 0.66 & 0.109 & 798 & 1.54 & 33 \\
O10a & 0.61 & 0.093 & 682 & 1.42 & 31 \\
O1 & 0.54 & 0.073 & 534 & 1.26 & 27 \\
O3a & 0.52 & 0.068 & 496 & 1.21 & 26 \\
O3 & 0.44 & 0.048 & 355 & 1.03 & 22 \\
O9a & 0.43 & 0.046 & 339 & 1.00 & 22 \\
O2 & 0.33 & 0.027 & 200 & 0.77 & 17 \\
O8a & 0.27 & 0.018 & 134 & 0.63 & 14 \\
O4 & 0.26 & 0.017 & 124 & 0.61 & 13 \\
\hline
\end{tabular}

The O-ring $\mathrm{O} 0$ is not sandblasted. The results are ordered after decreasing $w$

\subsection{Air Leakage Experiments and Theory}

The air leakage experiments was performed in Jülich using a home-made leakage set-up shown schematically in Fig. 13. In the actual experiments the steel plunger has two rubber O-rings (as in Fig. 11) in order to give a more stable (stiff) set-up, but the second O-ring has an axial cut (gas flow channel) and will not give any resistance to the gas or fluid flow but will contribute the to friction force acting on the plunger.

Let us apply the normal axial force $F_{x}=M g$ (where $M$ is the loading mass) to the plunger at time $t=0$ and assume all the air is removed at $t=t_{1}$, where the stopper has moved to the bottom $x=x_{1}$ of the barrel (i.e., reached the full air squeeze-out position) (see Fig. 13). The leakage experiment involves two phases. In the initial phase, from time $t=0$ to $t=t_{0}$, with typically $t_{0} \approx 10 \mathrm{sec}$, the stopper moves rapidly while compressing the air in the barrel. During this act the air leakage can be neglected so the number of gas molecules in the barrel equal

$N_{0}=\frac{p_{\mathrm{a}} V_{0}}{k_{\mathrm{B}} T}$,

where $V_{0}$ is the initial volume of trapped air at atmospheric pressure $p_{\mathrm{a}}$. During this rapid motion the air pressure in the barrel increases from $p_{\mathrm{a}}$ (the external pressure) to a higher pressure $p_{\mathrm{b}}^{*}$ while the gas volume change from $V_{0}$ to $V_{\mathrm{b}}^{*}$ given by

$V_{\mathrm{b}}^{*}=V\left(t_{0}\right)=V_{0}-x_{0} A_{\mathrm{b}}$,

where $x_{0}=x\left(t_{0}\right)$ and where $A_{\mathrm{b}}$ is the inner cross section area of the glass barrel. The pressure $p_{\mathrm{b}}^{*}$ is assumed to be given by the ideal gas law

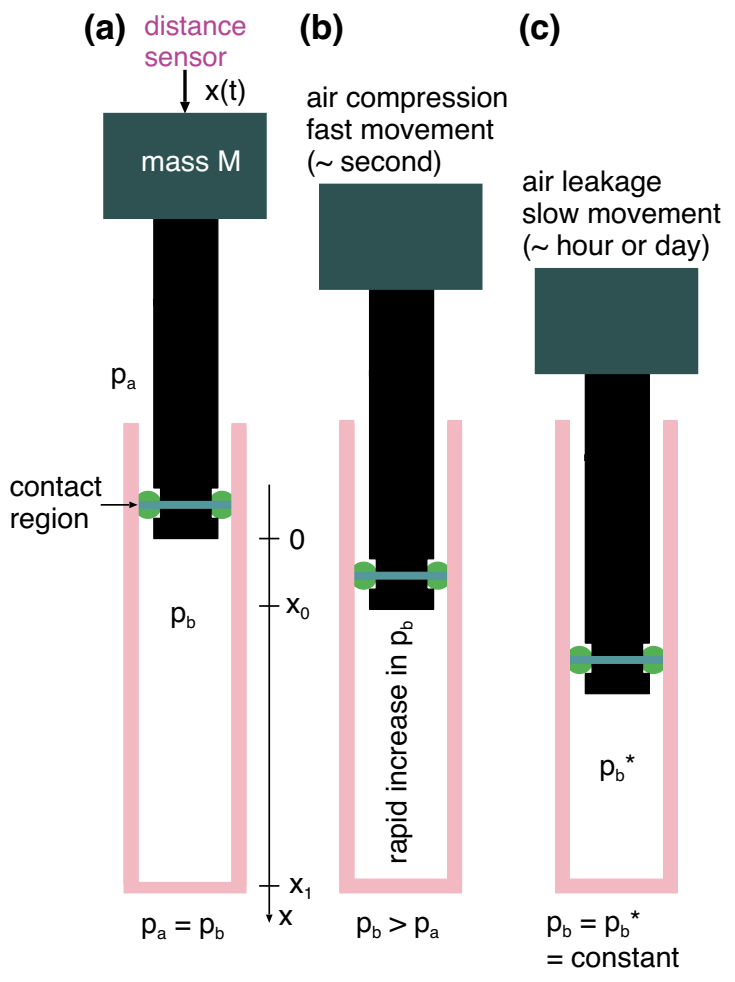

Fig. 13 The experimental set-up for measuring the air leakage rate (schematic)

$p_{\mathrm{b}}^{*} V_{\mathrm{b}}^{*}=N_{0} k_{\mathrm{B}} T$.

Assume that the properties of the glass barrel does not change with the lateral position $x$. In this case, after the initial fast compression of the gas, the pressure will remain at the value $p_{\mathrm{b}}^{*}$ while the stopper moves into the barrel with a constant speed $\dot{x}$ squeezing out the air from the barrel. The air leakage can be calculated by dividing the number of gas molecules removed, which equals $N_{0}$, by the time it takes, which equals $\Delta t=t_{1}-t_{0}$, giving

$\dot{N} \approx \frac{N_{0}}{\Delta t}$.

We can also calculate the friction force during the slow movement of the stopper using

$F_{\mathrm{f}}=F_{x}-\left(p_{\mathrm{b}}^{*}-p_{\mathrm{a}}\right) A_{\mathrm{b}}$.

We have found that usually the properties of the glass barrel varies slightly with the lateral position $x$. This may result from and $x$-dependency of the internal diameter of the glass barrel, or from variations in the rubber-glass frictional properties with $x$. This will result in a time-variation in the speed $\dot{x}$ of the stopper (or O-ring). In this case the gas pressure inside the barrel, and the friction force $F_{\mathrm{f}}$, will also depend on time (or $x$ ) even when $t>t_{0}$. This problem can be studied 
analytically too (see Appendix 1), but in what follows we will just present the average leakage rate obtained using (35).

\subsubsection{Experimental Air Leakage Results}

We have studied the leakage of air for a large number of O-rings with different surface roughness and different effective radius of the $\mathrm{O}$-ring (which depends on the time of sandblasting) and hence different width of the rubber-barrel contact region (see Figs. 11, 12 for some optical pictures of the contact region). In the experiments we applied an external axial force $F_{x}$ to the plunger which for the O-rings O1, O3-O5 was $F_{x}=2.2 \mathrm{~N}$ giving the pressure in the compressed air $p_{b}^{*} \approx 1.4$ bar as determined by the volume change during the initial fast air compression act (see Fig. 11b). For the O-rings O0, O2, O3a, O6a, O8a, O9a, O10a we used a bigger axial force $F_{x}=4.4 \mathrm{~N}$ giving the compressed air pressure $p_{b}^{*} \approx 2.2 \mathrm{bar}$. We note that in all cases the friction force $F_{\mathrm{f}}$ during the slow motion (typical velocity $\approx 1-10 \mu \mathrm{m} / \mathrm{s}$ ) during the air squeeze-out, is about $1 \mathrm{~N}$, and is mainly determined by the second (not sandblasted) O-ring (with an axial cut), which was the same in all the experiments.

Depending on the surface roughness and the contact pressure we observe that most of the O-rings do not leak (on the time scale of $\sim 20 \mathrm{~h}$ ) while O-rings O4, O8a and O10a leak (complete removal of the air in $\sim 1-20 \mathrm{~h}$ ). To illustrate this, in Fig. 14 we show the distance $x$ moved by the O-ring (or stopper) as a function of time for the not sandblasted O-ring O0 (red line) and O10a (blue line) with closed needle. For the O-ring O0, no air leakage occurred and the movement of the stopper (about $2 \mathrm{~cm}$ ) resulted in a compression of the air in the syringe so that the air pressure force $\left(p_{\mathrm{b}}^{*}-p_{\mathrm{a}}\right) A_{\mathrm{b}}$, and the friction force $F_{\mathrm{f}}$, just balance the applied force: $F_{x}=F_{\mathrm{f}}+\left(p_{\mathrm{b}}^{*}-p_{\mathrm{a}}\right) A_{\mathrm{b}}$ (where $A_{\mathrm{b}}$ is the inner barrel cross section).

Figure 15 shows similar results on a logarithmic time scale for a not sandblasted O-ring O0 (red line) and for O-ring O10a (blue line). For O-ring O10a the air pressure $p_{\mathrm{b}}^{*}$ in the barrel is 2.2 bar and the air leakrate $\dot{V}=1.3 \times 10^{-5} \mathrm{~cm}^{3} / \mathrm{s}$. The total measurement time in this experiment was $\approx 24 \mathrm{~h}$.

In Table 4 we summarize the results of all the measured air leakage rates, $\dot{Q}$ in $\mathrm{cm}^{3} / \mathrm{s}$, or equivalently mbar $\times$ liter / s. We also give the width of the rubber-glass contact region $w$ (in $\mathrm{mm}$ ), and the measured He leakrates for all the studied O-rings. For the air leakage for O-rings O1, O3-O5 the air pressure is $\approx 1.4$ bar inside the syringe and 1 bar outside, while for the O-rings O0, O2, O3a, O6a, O9a, O10a the air pressure is 2.2 bar inside the syringe and 1 bar outside. The glass barrels were cleaned to remove the silicone oil by immersing them in a bath of heptane for $12 \mathrm{~h}$, after which they where dried for a couple of days. For the O-rings where the leakage rates are

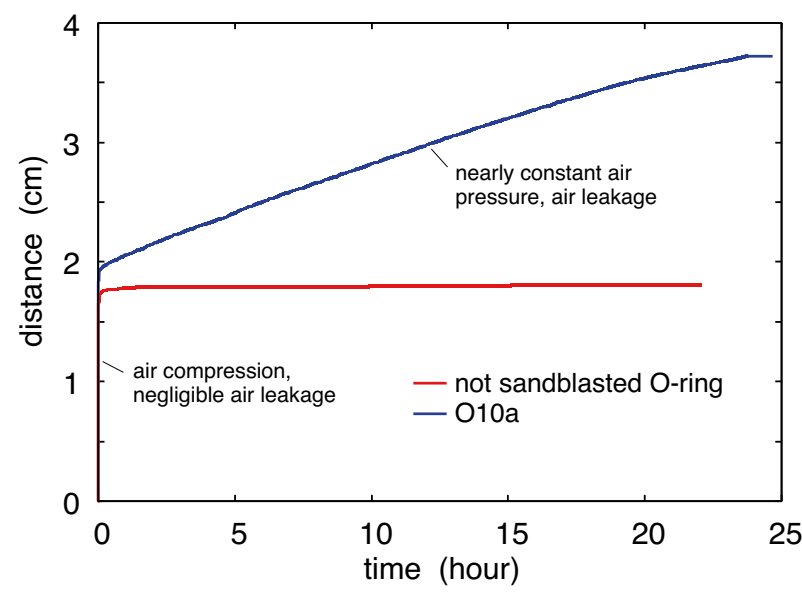

Fig. 14 The distance moved by the O-ring (or stopper) as a function of time for the O-ring $\mathrm{O} 0$ (red line) and O10a (blue line) with closed needle. The external (axial) force on the stopper is $F_{x}=4.4 \mathrm{~N}$ (Color figure online)

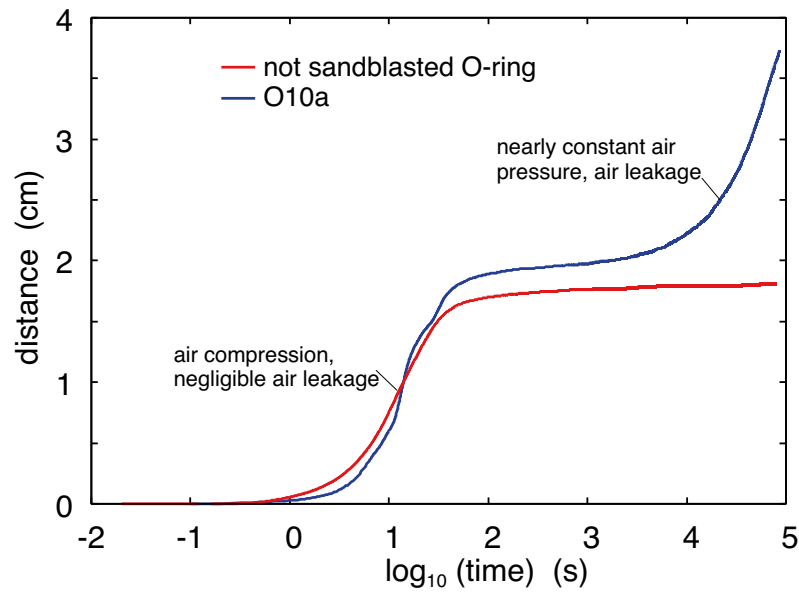

Fig. 15 The distance moved for O-ring O10a (blue) and for a not sandblasted O-ring O0 (red), as a function of the logarithm of time (Color figure online)

indicated as 0 , after the fast compression which occurs in typically $\sim 10 \mathrm{~s}$, we could not detect any movement of the stopper during a time period of at least $\sim 10 \mathrm{~h}$.

\subsubsection{Analysis of the Air Leakage Experiments}

Using the measured surface roughness power spectra we have calculated the air leakage rates for all the O-rings. In the calculations we have assumed the Hertz contact pressure (4) with the maximum pressure $p_{0}$ and the width $w$ given in Table 3. In the calculation of the pressure $p_{0}$ we have used the rubber modulus $E=7 \mathrm{MPa}$ (and Poisson ratio $v=0.5$ ) but for a linear viscoelastic material the calculated leakrate is in fact independent of the $E$-modulus because 
Table 4 The width of the rubber-glass contact region $w$ (in $\mathrm{mm}$ ) in the air and $\mathrm{He}$ leakage experiments, and the measured $\mathrm{He}$ and air leakrate $\dot{Q}$ (in $\mathrm{cm}^{3} / \mathrm{s}$ or equivalently mbar $\times$ liter $/ \mathrm{s}$ ) for all studied O-rings

\begin{tabular}{lllll}
\hline O-ring & $\begin{array}{l}w \text { (air) } \\
(\mathrm{mm})\end{array}$ & $\begin{array}{l}w(\mathrm{He}) \\
(\mathrm{mm})\end{array}$ & $\begin{array}{l}\dot{Q} \text { (air) } \\
\mathrm{cm}^{3} / \mathrm{s}\end{array}$ & $\begin{array}{l}\dot{Q}(\mathrm{He}) \\
\mathrm{cm}^{3} / \mathrm{s}\end{array}$ \\
\hline O0 & 1.25 & 1.11 & 0 & 0 \\
O5 & 0.75 & 0.59 & 0 & $(0 ; 0)$ \\
O6a & 0.66 & 0.69 & 0 & $6.5 \times 10^{-6}$ \\
O10a & 0.61 & 0.72 & $1.3 \times 10^{-5}$ & $1.2 \times 10^{-5}$ \\
O1 & 0.54 & - & 0 & - \\
O3a & 0.52 & 0.52 & $(0 ; 0)$ & $1.0 \times 10^{-5}$ \\
O3 & 0.44 & 0.49 & 0 & 0 \\
O9a & 0.43 & 0.40 & 0 & 0 \\
O2 & 0.33 & 0.35 & $(0 ; 0)$ & 0 \\
O8a & 0.27 & 0.23 & $(3.3 ; 2.1 ; 1.2) \times 10^{-3}$ & $5.0 \times 10^{-3}$ \\
O4 & 0.26 & 0.26 & $3.2 \times 10^{-5}$ & $7.0 \times 10^{-5}$ \\
\hline
\end{tabular}

The results are ordered after decreasing $w$. For the He leakage the He pressure is 1 bar inside the syringe and 0 bar outside. For the air leakage for O-rings O1, O3-O5 the air pressure is $p_{\mathrm{b}}^{*} \approx 1.4$ bar inside the syringe and 1 bar outside. For the air leakage O-rings O2, O3a, O6a, O9a, O10a the air pressure is 2.2 bar inside the syringe and 1 bar outside. The glass barrels were cleaned to remove (most of) the silicone oil. For the not sandblasted O-ring $\mathrm{O} 0$ the air leakage experiment does not detect any leakage over a time period of $24 \mathrm{~h}$. The He leakage experiment for the same system gave $5.1 \times 10^{-7} \mathrm{~cm}^{3} / \mathrm{s}$ which we do not attribute to true leakage at the rubber-barrel interface but to some other source of He atoms, e.g., desorption of He atoms from the camber wall. All He leakage rates below $10^{-6} \mathrm{~cm}^{3} / \mathrm{s}$ are indicated by 0 in the table as they may be due to diffusion of He through the rubber matrix or other effects not related to interfacial He leakage. When several leakage rates are given for the same system it is due to repetitions of the experiments

the interfacial separation $u(x, y)$ does not depend on the $E$-modulus when the compression $\delta$ is given, as is the case for an O-ring confined inside the barrel. Thus, the width $w$ is determined by the radius $R$ and the compression $\delta$ (as $\left.w=(4 R \delta)^{1 / 2}\right)$ and does not depend on the $E$-modulus. The maximum pressure $p_{0}$ does increase linearly with increasing $E$-modulus but the separation at the interface depends on $p / E$ and is hence independent of the $E$-modulus. For real rubber materials, which exhibit strain softening for small strain (and strain-stiffening for large strain), this results holds accurately only if the strain and hence the effective modulus is similar in the macroscopic Hertz contact region as in the asperity contact region. The macroscopic strain is typically $10-60 \%$ (see Table 3) while the strain in the asperity contact regions may be slightly larger but for large strain the strain softening does not vary strongly with the strain level.

Table 4 shows that for O-rings O0-O3, O5, O3a, O6a and O9a we did not observe any air leakage. This is in agreement with our calculated leakrates. Thus in Fig. 16 we show the calculated square-unit leakrate for O-ring $\mathrm{O} 1$ (pink), $\mathrm{O} 3$

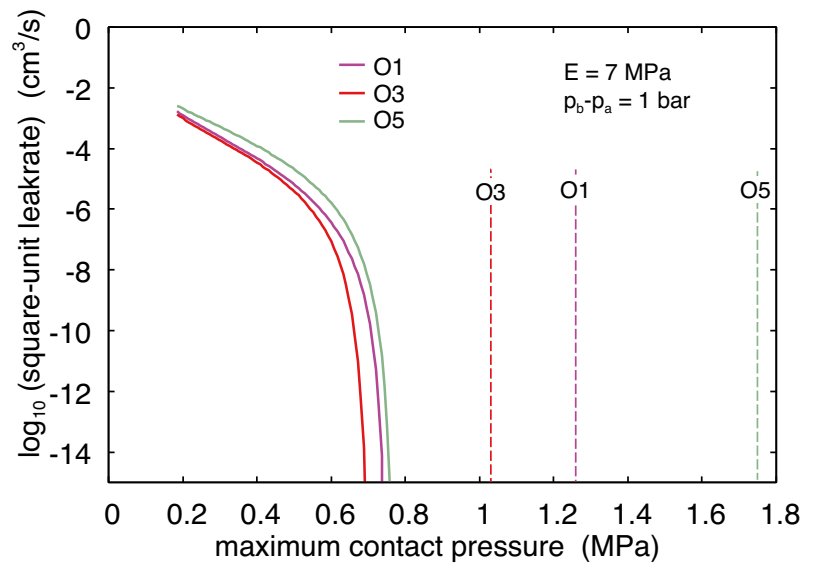

Fig. 16 The calculated square-unit leakrate for O-ring O1 (pink), O3 (red) and $\mathrm{O} 5$ (gray) as a function of the maximum pressure in the Hertz contact region. The vertical dashed lines indicate the actual maximum pressures ( $p_{0} \approx 1.26,1.03$ and $1.75 \mathrm{MPa}$, respectively) in the rubber-glass contact region. The theory predict no gas leakage for these O-rings which agree with observations (Color figure online)

(red) and O5 (gray) as a function of the maximum pressure in the Hertz contact region. In the calculation we have used the measured surface roughness power spectra of the O-rings. The vertical dashed lines indicate the actual maximum pressures $p_{0} \approx 1.26,1.03$ and $1.75 \mathrm{MPa}$, respectively (see Table 3), obtained from the width $w$ of the contact region. In the calculation we have assumed that the air pressure in the syringe is 2 bar and 1 bar outside the syringe, but the leakage for any other pressure difference $\Delta p$ can be obtained by direct scaling since the ballistic contribution to the leakrate is proportional to $\Delta p$. Clearly, at the maximum pressure in the Hertz contact region the contact area percolate in all cases, and the leakage rate vanish.

For the O-ring $\mathrm{O} 4$ we do observe leakage with $\dot{Q}=3.2 \times 10^{-5} \mathrm{~cm}^{3} / \mathrm{s}$. In Fig. 17 we show the calculated leakrate $\dot{Q}$ for O-ring O4 (green line) as a function of the maximum pressure $p_{0}$ in the Hertz contact region. In the calculation we have used the measured surface roughness power spectrum of the O-ring O4 (the 1D power spectra are shown in Fig. 8, and was converted into the 2D power spectra assuming isotropic roughness). The vertical dashed line indicate the actual maximum pressures $p_{0} \approx 0.61 \mathrm{MPa}$ in the contact region for O-ring $\mathrm{O} 4$ in the glass barrel, as calculated using the Hertz theory (see Table 3 ). In the calculation we have used the (measured) air pressure in the syringes, namely 1.52 bar for O-ring $\mathrm{O} 4$, and 1 bar outside the syringe. The leakrate assuming this pressure is $\approx 3.4 \times 10^{-5} \mathrm{mbar} \times 1 / \mathrm{s}$ for O-ring $\mathrm{O} 4$, in good agreement with the measured leakrate.

In Fig. 18 we show the surface separation $u_{\mathrm{c}}$ at the critical constrictions for O-ring $\mathrm{O} 4$ as a function of the maximum pressure $p_{0}$ in the Hertz contact region. The vertical 


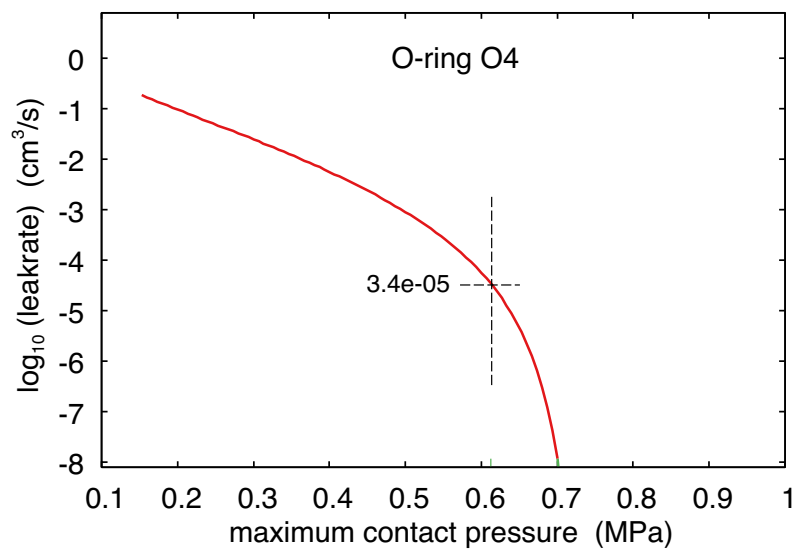

Fig. 17 The calculated leakrate $\dot{Q}$ for O-ring $\mathrm{O} 4$ as a function of the maximum pressure $p_{0}$ in the Hertz contact region

dashed lines indicate again the actual maximum pressure $p_{0} \approx 0.61 \mathrm{MPa}$ in the contact region for the O-ring $\mathrm{O} 4 \mathrm{in}$ the glass barrel. The surface separation $u_{\mathrm{c}}$ for the $\mathrm{O} 4 \mathrm{O}$-ring is about two times smaller than the air molecule mean free path, and the air leakage is mainly ballistic.

Figure 19 shows the logarithm of the calculated air leakage rate as a function of the maximum pressure in the Hertz contact pressure distribution for O-rings O3a, O8a and O10a. The vertical dashed lines indicate the contact pressures calculated from the width $w$ of the contact region $\left(p_{0}=1.17,0.54\right.$ and $\left.1.69 \mathrm{MPa}\right)$. For the O-rings O8a and $03 \mathrm{a}$ the predicted leakage rates $\left(2.5 \times 10^{-3} \mathrm{~cm}^{3} / \mathrm{s}\right.$ and $\approx 0$, respectively) are close to the observed leakage rates $(1.2-3.3) \times 10^{-3} \mathrm{~cm}^{3} / \mathrm{s}$ and 0 , respectively. For the O-ring $\mathrm{O} 10 \mathrm{a}$ the predicted leakage rate is smaller than observed,

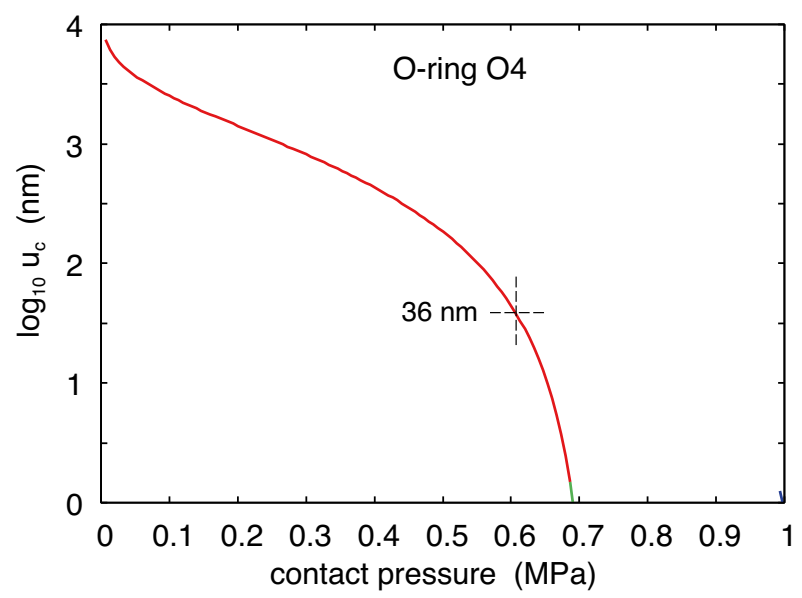

Fig. 18 The surface separation $u_{\mathrm{c}}$ at the critical constrictions for O-ring $\mathrm{O} 4$ as a function of the maximum pressure $p_{0}$ in the Hertz contact region. The vertical dashed lines indicate the actual maximum pressure $p_{0} \approx 0.61 \mathrm{MPa}$ in the rubber-glass contact region, as calculated using the Hertz theory (see Table 3 ) but a relative small change in the contact pressure (from $1.69 \mathrm{MPa}$ to $\approx 1.5 \mathrm{MPa}$ ) would give agreement between the calculated and measured leakage rates.

The agreement found above between theory and experiment is very good, in particular taking into account that the width $w$ measured optically is not highly accurate due to blurred nature of the contact line (due to the surface roughness, the curved nature of the glass barrel). The surface roughness power spectrum used in the calculations has also some uncertainty. To illustrate this, in Fig. 20 we show the logarithm of the calculated air leakage rate as a function of the maximum Hertz contact pressure for O-ring O3a. Results are shown using the power spectra of the 7 line tracks, each 2 $\mathrm{mm}$ long (thin lines) and using the average power spectrum (thick line) shown in Fig. 10.

We have found that using glass barrels from different batch can have a relative large influence on the leakage rate. We attribute this to small changes in the internal diameter of the glass barrel. Because of the small compression (or penetration) for some rubber O-rings, e.g., only $\delta=17 \mu \mathrm{m}$ for the O-ring $\mathrm{O} 4$ (see Table 3), the variation in the internal diameter specified by the tolerance $\pm 100 \mu \mathrm{m}$ can result in non-contact in some case. However, typical variation within a batch of produced syringes is much smaller and using glass barrels from the same batch gives reproducible results. This is illustrated in Fig. 21 which shows the displacement of the rubber stopper in measurements for O-ring O9a in two different glass barrels from the same batch.

\subsection{Helium Leakage Experiments}

We have performed Helium (He) leakage experiments with the PFEIFFER Vacuum ASM340. The schematic set-up is shown in Fig. 22. The region outside of the syringe on the

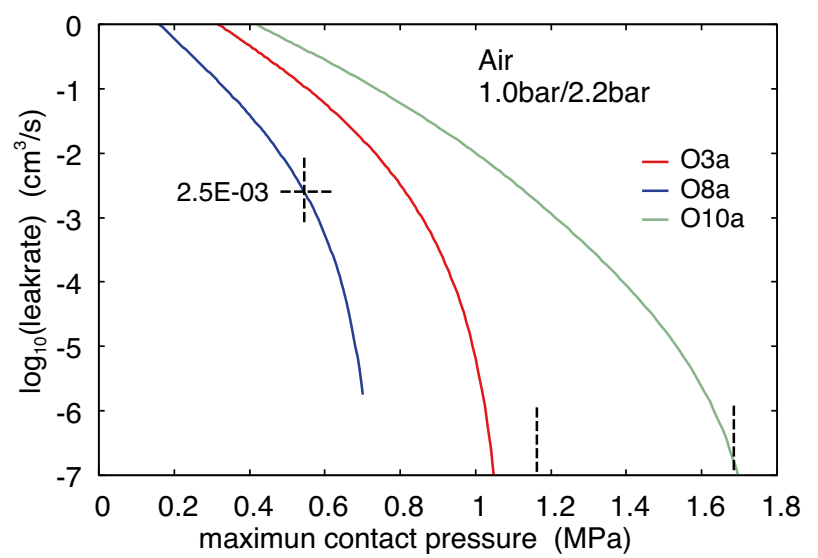

Fig. 19 The logarithm of the calculated air leakage rate as a function of the maximum pressure in the Hertz contact pressure distribution for O-rings O3a, O8a and O10a. In the experiment the air pressure in the syringes is 2.2 bar and outside 1 bar 


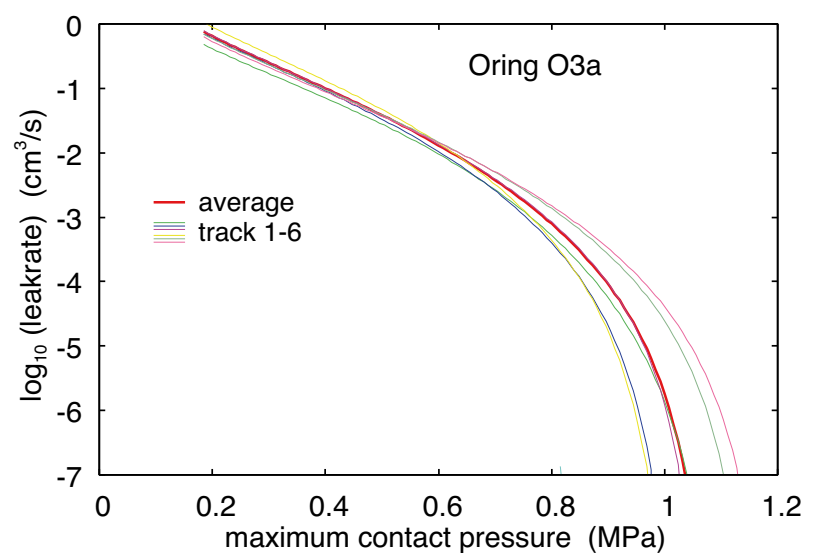

Fig. 20 The logarithm of the calculated air leakage rate as a function of the maximum pressure in the Hertz contact pressure distribution for O-ring O3a. Results are shown using the power spectra of 7 line tracks each $2 \mathrm{~mm}$ long (thin lines) and using the average power spectrum (thick line) shown in Fig. 10

plunger side is located in a volume connected to a vacuum pump and a He leak detector. The He detector (a mass spectrometer) monitors the He gas concentration. After the syringe is placed in the chamber, in order to remove (or reduce) the He adsorbed on the solid walls (which are in kinetic equilibrium with the He gas in the chamber) the vacuum pump keep a vacuum for at least $1 \mathrm{~h}$. Once the chamber is decontaminated, He is introduced in the syringe, and the mass spectrometer detector monitors the leakage of $\mathrm{He}$ through the rubber stopper. However, even for a not sandblasted rubber O-ring a finite He leak current is observed at the rate $\dot{Q}(\mathrm{He}) \approx 5 \times 10^{-7} \mathrm{mbar} \cdot 1 / \mathrm{s}$ which must result from other He leakage sources, e.g., diffusion of He through the rubber or from $\mathrm{He}$ adsorbed on the inner walls of the test equipment. Hence, in the present set-up leakrates less than

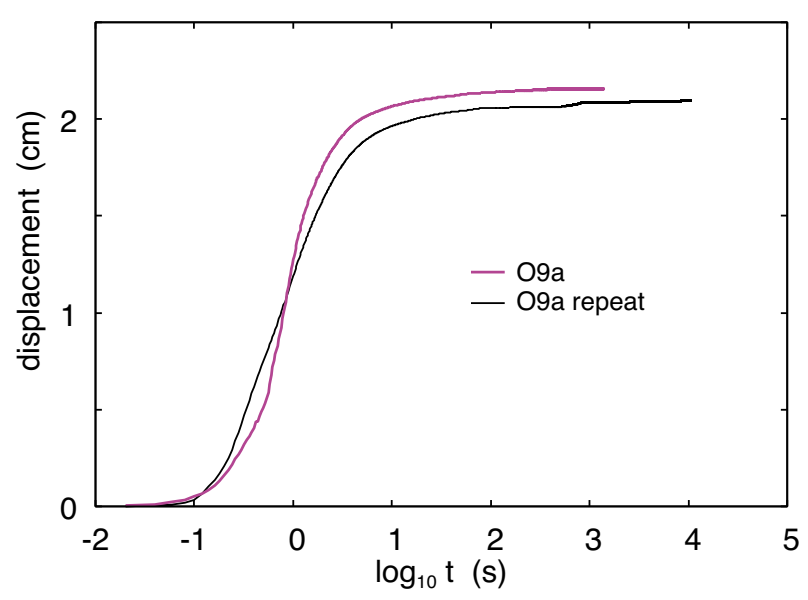

Fig. 21 The displacement of the rubber stopper in measurements for $\mathrm{O}$-ring $\mathrm{O} 9 \mathrm{a}$ in two different glass barrels from the same batch

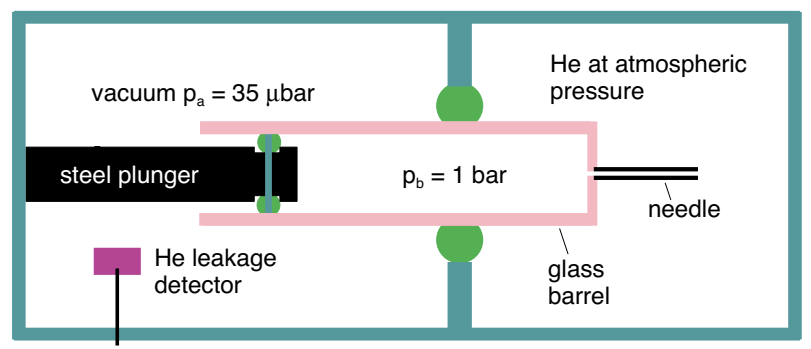

Fig. 22 Experimental set-up for measuring He gas leakage (schematic). The He detector is a mass spectrometer

$\dot{Q}(\mathrm{He}) \approx 10^{-6} \mathrm{mbar} \cdot 1 / \mathrm{s}$ may occur even when there is no true interfacial He leakage.

In Table 4 we summarize the results of all the measured leakage rates for $\mathrm{He}$ (and air). We also give the width of the rubber-glass contact region $w$. For the three $\mathrm{O}$-rings $\mathrm{O} 4, \mathrm{O} 8 \mathrm{a}$ and O10a where we observe air leakage we observe similar $\mathrm{He}$ leakage rates. The $\mathrm{He}$ leakage rate for O-ring $\mathrm{O} 4$ and $\mathrm{O} 8 \mathrm{a}$ is about $\sim 2$ times higher than in air which may be due to the roughly $\sim 2.6$ times higher average velocity $\bar{v}$ of He atoms at room temperature as compared to air molecules (note: the leakage rate is proportional to $\bar{v}$ in the ballistic limit, see (30)). For O-rings O3, 05 and $\mathrm{O} 9 \mathrm{a}$ the He leakage is below $10^{-6} \mathrm{~cm}^{3}$ which we do not attribute to interfacial glass leakage and with therefore are denoted as zero leakage rate in Table 4, which agree with the air leakage observation. For the O-rings O3a and O6a we observe no air leakage but the He leakage rates are of order $10^{-5} \mathrm{~cm}^{3}$.

\subsection{Water Leakage Experiments}

We have performed leakage experiments for syringes filled with water using the Jülich experimental set-up. We considered two cases where the glass barrel is either completely filled with water or where an air bubble exist between the inner rubber O-ring and the water. Figure 23 shows the displacement of the stopper as a function of time for a water filled glass barrel for O-ring $\mathrm{O} 4$. For $t>5 \mathrm{~h}$ the plunger moves with a nearly constant speed $\approx 1 \mu \mathrm{m} / \mathrm{h}$ corresponding to a very small leakage rate, $\approx 5 \times 10^{-9} \mathrm{~cm}^{3} / \mathrm{s}$. We note that if the air leakage for the unfilled (dry) barrel would be due to viscous flow of the air, then one would expect the water leakage to be (roughly) smaller than the air leakage by a factor of $\approx \eta_{\text {air }} / \eta_{\text {water }}$ where $\eta_{\text {air }} \approx 2 \times 10^{-5} \mathrm{~Pa}$ s is the viscosity of air and $\eta_{\text {water }} \approx 10^{-3} \mathrm{Pas}$ the viscosity of water. In reality, the air flow is mainly ballistic and the change in the leakrate when going from air to water is much larger $\approx 856$ i.e., much bigger than the factor of 50 expected if the air flow would be viscous. The air leakage rate (see Table 4) is $\approx 3.2 \times 10^{-5} \mathrm{~cm}^{3} / \mathrm{s}$ so we expect for water the leakage rate $\approx(3.2 / 856) \times 10^{-5} \approx 4 \times 10^{-8} \mathrm{~cm}^{3} / \mathrm{s}$ which is 


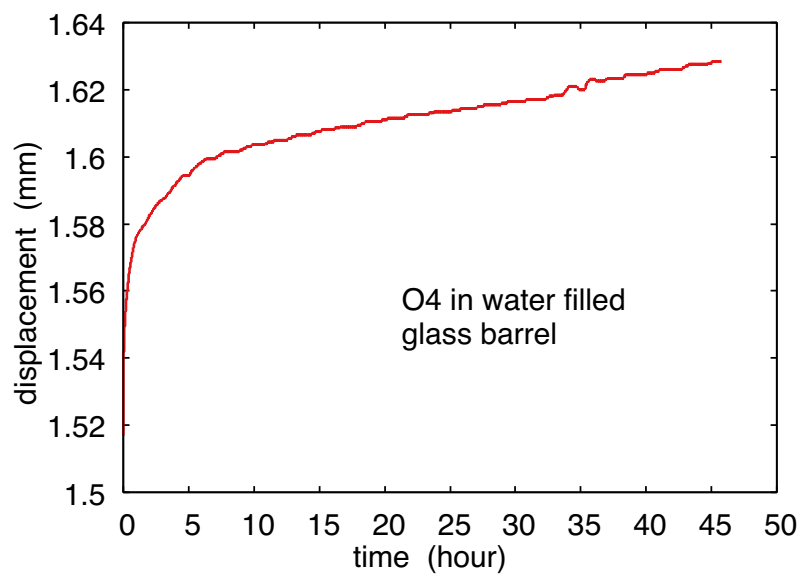

Fig. 23 The displacement of the stopper as a function of time for a water filled glass barrel for O-ring $\mathrm{O} 4$

nearly 10 times bigger than the observed leakage rate. In fact the small movement observed (of order $30 \mu \mathrm{m}$ during $40 \mathrm{~h}$ ) could be due to viscous relaxation of the rubber O-ring due to the axial force, or due to dissolution of small air bubbles.

Figure 24 shows the O-ring $\mathrm{O} 4$ in (a) a dry glass barrel and (b) in a water filled barrel and (c) in a water filled barrel with air bubble close to the O-ring. The rubber-glass contact region for the barrels with water must be the same as for the dry glass barrel in (a) but due to the influence of the water on the optical picture it appears as if the contact is much wider but this is an optical illusion. The important point is that for the water filled barrel with an air bubble, a water capillary bridge occurs between the rubber stopper and the glass barrel resulting in an optical picture very similar to the case of a completely water filled barrel.

Figure 25 shows schematically two barrel filled with water where in (a) an air bubble remains at the top close to the rubber stopper while in (b) the glass barrel is completely filled with water.

To explain why no (water or air) leakage may occurs for the barrel partly or completely filled with water, note that if the interface is hydrophobic in water a finite pressure is needed to squeeze the fluid through the critical junction (see Fig. 26a). For an hydrophobic interface the (advancing) water contact angles $\theta$ and $\theta^{\prime}$ on the two solid walls (here rubber and silica glass which may be covered by one or a few monolayers of silicon oil) must satisfy $\cos \theta+\cos \theta^{\prime}<0$. The fluid pressure

$p=-\left(\cos \theta+\cos \theta^{\prime}\right) \frac{\gamma}{u}$

where $u=u(x, y)$ is the surface separation and $\gamma \approx 0.07 \mathrm{~J} / \mathrm{m}^{2}$ the water surface tension. Using $u=100 \mathrm{~nm}$ we get $\gamma / u \approx 10 \mathrm{MPa}$. In the present case the water pressure is $\approx 0.1 \mathrm{MPa}$. Hence if $-\left(\cos \theta+\cos \theta^{\prime}\right)>0.01$ it is impossible
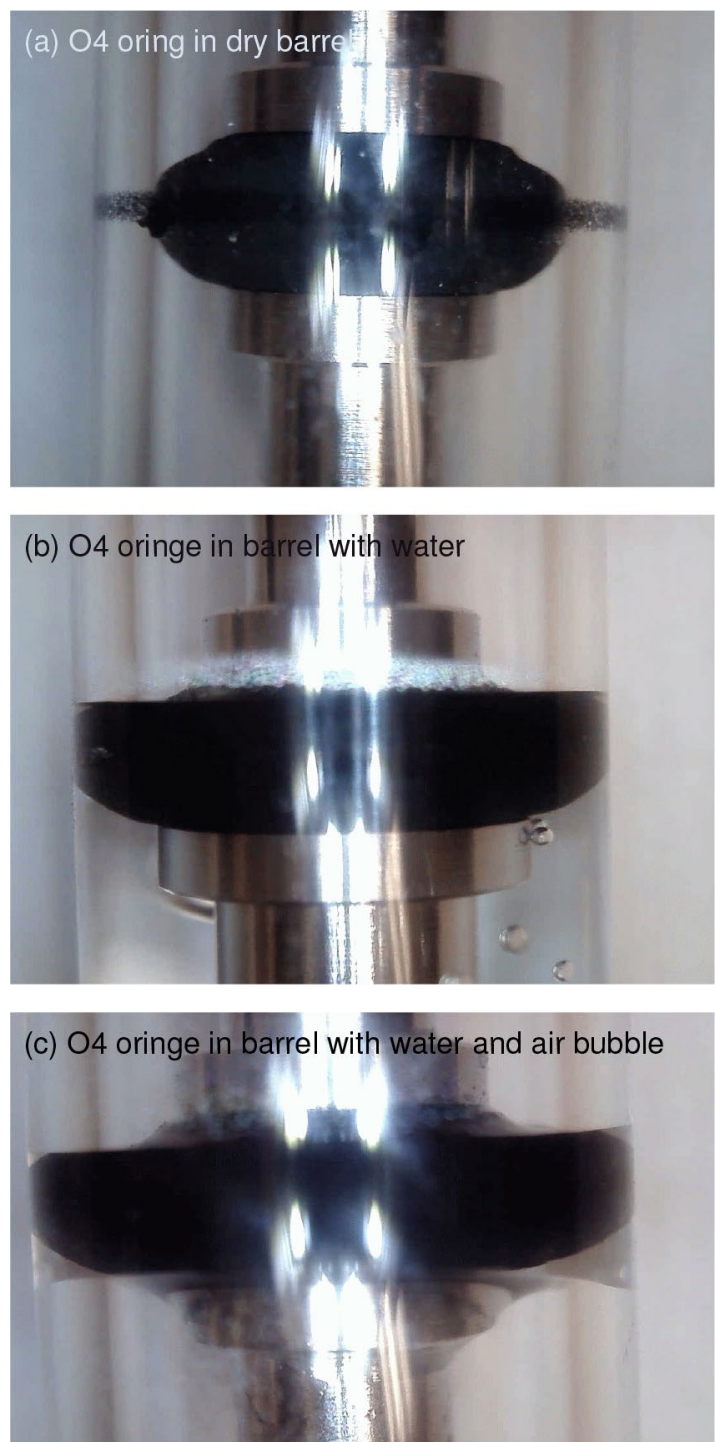

Fig. 24 O-ring $\mathrm{O} 4$ in a dry glass barrel, $\mathbf{b}$ water filled barrel and c water filled barrel with air bubble close to the O-ring. In case $\mathbf{c}$ a water capillary bridge exist at the O-ring

to squeeze the fluid through the critical junction, as indeed observed. For an hydrophilic interface it may be impossible to remove a fluid capillary bridge but now the resistance to squeeze-out comes from the exit side of the critical junction (and involves the receding contact angles) rather than on the entrance side as expected for a hydrophobic interface, see Fig. 26b. If the rubber stopper would be surrounded by water on both sides and if no air bubble would exist, then there would be no capillary effects.

\subsection{Discussion}

It would have been interesting to correlate the observed leakrates with optical pictures of the rubber-glass contact 


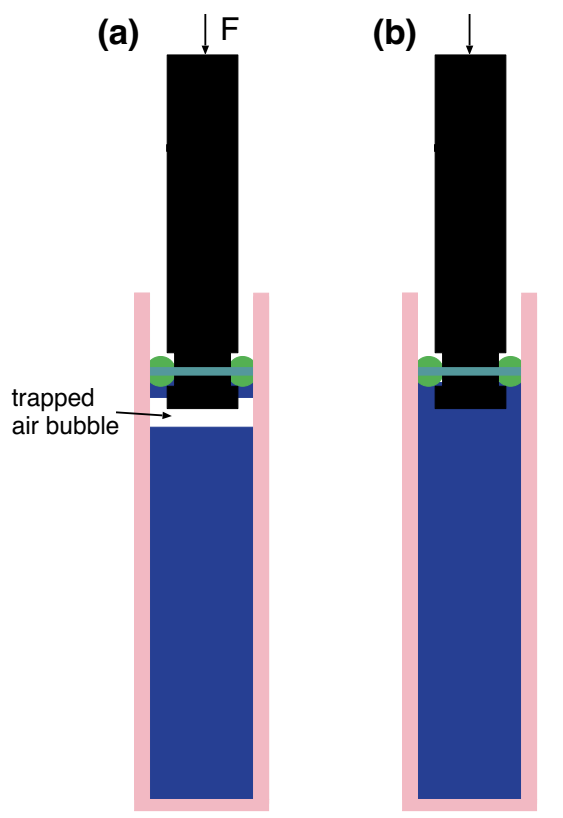

Fig. 25 Glass barrel filled with water. In a an air bubble remains at the top close to the rubber stopper. In addition a water capillary bridge occurs at the rubber O-ring. In $\mathbf{b}$ the glass barrel is filled with water to the rubber O-ring i.e., no air bubble exist

(a) hydrophobic interface

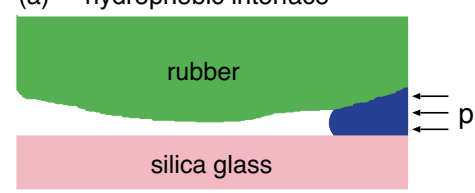

(b) hydrophilic interface

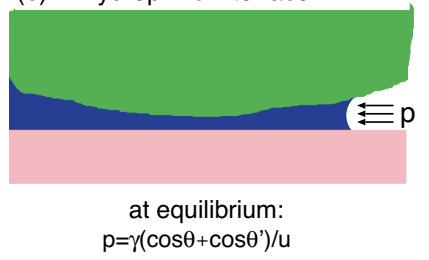

Fig. 26 a For a hydrophobic interface a finite fluid pressure is needed to squeeze the fluid through the critical junction. $\mathbf{b}$ For a hydrophilic interface a finite fluid pressure is needed to remove a fluid capillary bridge from the critical junction

region. Unfortunately, because of the curved nature of the interface and the finite resolution of the optical microscopes it is not possible to quantitatively determine the relative area of real contact $A / A_{0}$ which would have been of great interest as the theory predict no fluid leakage when the area of real contact percolate, which from theory (in the absence of adhesion) is expected when $A / A_{0} \approx 0.42$. Still for the sandblasted O-rings the optical pictures shows that the contact is incomplete (see Figs. 24 and 27).
When the stopper is pushed into a syringe (with closed needle) compressed air form in the barrel. Thus the rubber O-ring is exposed to an axial air pressure force in one direction and an opposite axial force from the plunger rod. As a result they contract a little in the axial direction and since rubber is nearly incompressible, it tends to expand in the radial direction. This will increase the rubber-glass contact pressure and hence reduce the leakrate. For the cases studied above the axial force is relative small and has only a small influence on the leakrate. However, we performed experiments with a much bigger axial force, about $15 \mathrm{~N}$, then used before, and made an interesting observation (see Fig. 28): When inserted in the glass barrel with a small axial force a sandblasted O-ring had the rubberglass contact width $\approx 0.3 \mathrm{~mm}$. If the squeezing force is increased to $15 \mathrm{~N}$ (giving an air pressure of about 5 bar) then the width of the rubber-glass contact region becomes $\approx 0.6 \mathrm{~mm}$ i.e., about double of what is obtained when there is no applied squeezing force. This imply that the (maximum) rubber-glass contact pressure increases with increasing axial force. In Hertz theory for cylinder against a flat the maximum pressure $p_{\max }=E^{*} w /\left(4 r_{0}\right)$ where $w$ is the width of the contact region and $r_{0}$ the inner radius of
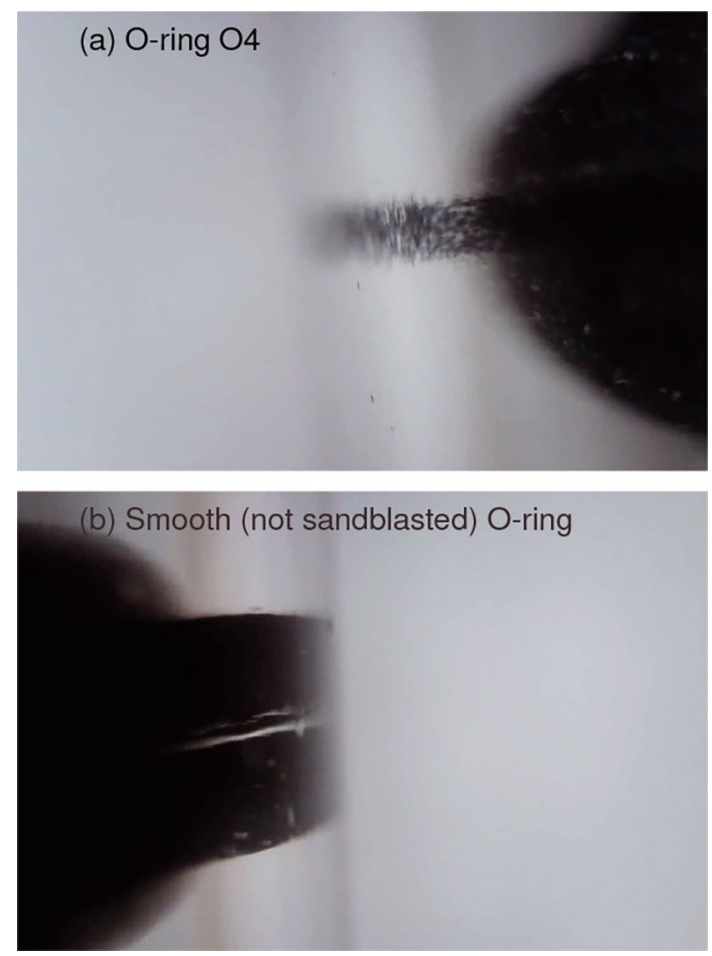

Fig. 27 The contact region between a the sandblasted O-ring $\mathrm{O} 4$ and the glass barrel, and $\mathbf{b}$ a not sandblasted (smooth) O-ring and the glass barrel. Note the incomplete contact in (a) and the apparent complete contact for the smooth O-ring in (b), except for a strip (white line) in the middle of the contact due to extra material (flash) formed at the parting lines in the mold (the flash disappear upon sandblasting) 
the glass barrel. Hence increasing the width with a factor of 2 will increase the maximum pressure by a factor of 2 . In the present case this will result in a percolation of the contact area in the central area of the rubber-glass contact area. Hence no gas leakage is expected when the axial squeezing force equal $15 \mathrm{~N}$. This is in qualitative accordance with our observations. When we remove the axial force the stopper retract some distance and the width of the rubber-glass contact region (nearly) return to the original value found when the axial force vanish. At the same time a "puff" sound can be heard. If axial force is immediately returned to the original value $(15 \mathrm{~N})$ the volume of compressed air is much smaller. Clearly, during the short time period where the axial force was removed, a considerable amount of air was leaking through the rubber O-ring seal. This may in part be due to the air pressure helping to separate the rubber from the glass in the nominal contact area.

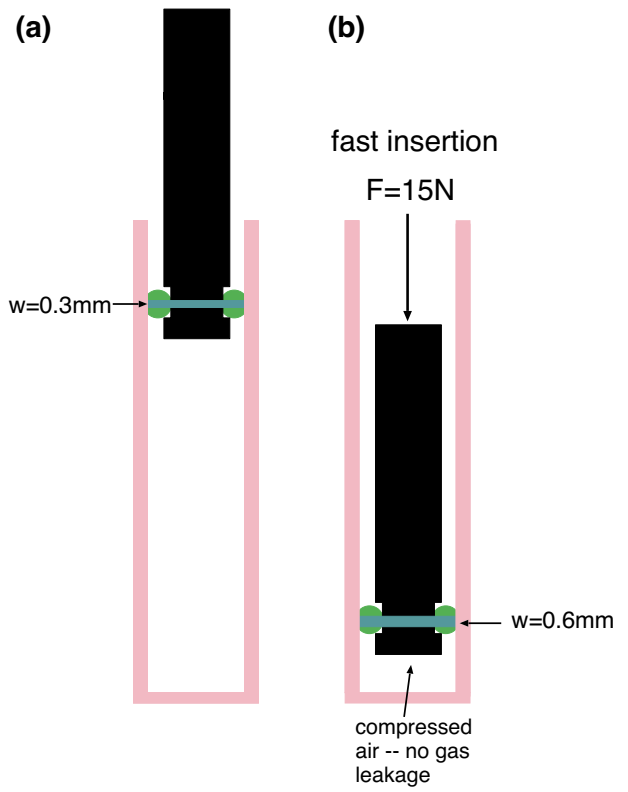

(c)

fast gas leakage or removal of force $\mathrm{F}$

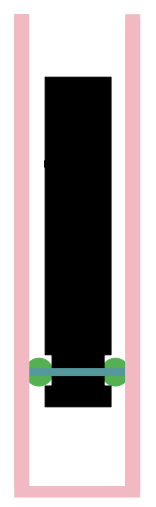

Fig. 28 a When the stopper is inserted slowly in the barrel the rubber-glass contact region has a width $w \approx 0.3 \mathrm{~mm}$. If it moves slowly towards the top of the barrel the air leak out and the width of the rubber-glass contact region stays at the value $w \approx 0.3 \mathrm{~mm}$ during the whole insertion process. $\mathbf{b}$ If the stopper is inserted fast, with a big axial force $F \approx 15 \mathrm{~N}$, the rubber-glass contact width increases to $w \approx 0.6 \mathrm{~mm}$ while a region of compressed air forms between the O-ring and the top of the barrel. In this state no air leakage is observed indicating that the rubber-barrel contact pressure is so high that the contact area percolate and no open non-contact channels occur at the rubber-glass interface. (c) If the applied axial force is removes the stopper retract some distance and the width of the contact region return to its original value $w \approx 0.3 \mathrm{~mm}$. If the external force is applied again the stopper moves much closer to the top of the barrel indicating that air has leaked out during the time period when the axial force vanished

\section{Summary and Conclusions}

A theory was developed for the leakage of gases at the interface between elastic solids with random roughness. A simple equation was derived for the gas pressure drop over narrow junctions (critical constrictions), which takes into account the gas molecule mean free path, and the interfacial separation between the solids. Using this equation and the Bruggemann effective medium theory for the fluid flow conductivity, and the Persson's contact mechanics theory for the probability distribution of interfacial separation, we predicted gas leakrates in good agreement with experiments. We applied the theory to gas and water leakage for syringes with glass barrels and rubber O-ring stoppers. The O-rings were sandblasted to generate well-defined random roughness, resulting in open (percolating) flow channels in the glass-rubber nominal contact region. We studied the leakage of helium, air and water by applying a pressure difference between inside and outside the syringe barrel. For gases (helium and air) the leakage involved ballistic motion of the gas molecules as mean free path of the gas molecules was of order, or larger than, the mean interfacial separation in the most narrow junctions along the open flow channels. The theory agreed very well with experimental results for leakage of gases. For water we observed no leakage which we attribute to surface energy effects. Thus the applied pressure was not big enough to overcome the Laplace pressure. We have shown that for (linear) viscoelastic materials the leakrate is independent of the effective $E$-modulus because the interfacial separation $u(x, y)$ does not depend on the $E$-modulus when the compression $\delta$ is given, as is the case for a rubber O-ring when confined between the syringe plunger road and the barrel.

\section{Appendix 1}

The friction force between the rubber O-rings and the glass barrel change as a function time or sliding distance. During the initial short time period $t_{0}$, where the stopper moves from $x=0$ to $x=x\left(t_{0}\right)=x_{0}$, the air in the barrel get compressed and the air pressure change from $p_{\mathrm{a}}$ to the final value $p_{\mathrm{b}}^{*}$. During this rapid act there is negligible air leakage so that neglecting inertia (which is a good approximation except for the first few seconds) the friction force

$F_{\mathrm{f}}(t)=F_{x}-\left(p_{\mathrm{b}}(t)-p_{\mathrm{a}}\right) A_{\mathrm{b}}$

where, assuming that the temperature is constant, the ideal gas law gives $p_{\mathrm{b}}(t)=p_{\mathrm{a}} V_{0} / V(t)$, where $V_{0}=x_{1} A_{\mathrm{b}}$ is the initial volume and $V(t)=V_{0}-x(t) A_{\mathrm{b}}$. For times $t>t_{0}$ the friction force is given by $F_{\mathrm{f}}=F_{x}-\left(p_{\mathrm{b}}^{*}-p_{\mathrm{a}}\right) A_{\mathrm{b}}$ and would 
be independent of time if the glass barrel properties (e.g., inner diameter) would be the same along the whole syringe. We have found that the later assumption is not strictly true and the velocity of the stopper and hence the friction force depend on time for $t>t_{0}$. As an example in Fig. 29 we show the friction force for the O-ring $\mathrm{O} 2$ as a function of the sliding speed. There are two different regions in the figure denoted A (red curve) and B (blue). The first highvelocity region $\mathrm{A}$ is the friction experienced by the stopper during the first fast compression act (not including the initial $3 \mathrm{~s}$ where inertia is important $)\left(t<t_{0} \approx 100 \mathrm{~s}\right)$. The noisy region $B$, where the sliding speed is of order $10 \mu \mathrm{m} / \mathrm{s}$, is the slow squeeze-out region extending from $t=t_{0}$ to $t=t_{1} \approx 3000 \mathrm{~s}$. The friction force in this slow motion region varies from $0.8 \mathrm{~N}$ to $1.2 \mathrm{~N}$ indicating some variation in the barrel properties in the axial direction. Here the time-variation of the friction force was obtained assuming that the air leakrate is given by $\beta\left(p_{\mathrm{b}}^{*}-p_{\mathrm{a}}\right)$ where $\beta$ is a constant but where $p_{\mathrm{b}}^{*}(t)$ fluctuate slightly in time as determined by the time dependency of $x(t)$. If the changes in the friction force is due to variations in the internal diameter of the glass barrel, then it is likely that there will also be fluctuations in the leakrate coefficient $\beta(t)$ but the present approach is not able to determine the time variations of $\beta$ and $p_{\mathrm{b}}^{*}$ separately, but this does not change the main conclusion. (The variation of the friction force with the position of the stopper in the glass barrel could have been studied directly by performing measurements on syringes with open needle where there is no air pressure force term. However, no such study was performed.) We have observed similar magnitude $\sim 1 \mathrm{~N}$ of the friction force for the other O-rings, which is expected as the largest contribution to the friction force probably arises from the second not sandblasted O-ring.

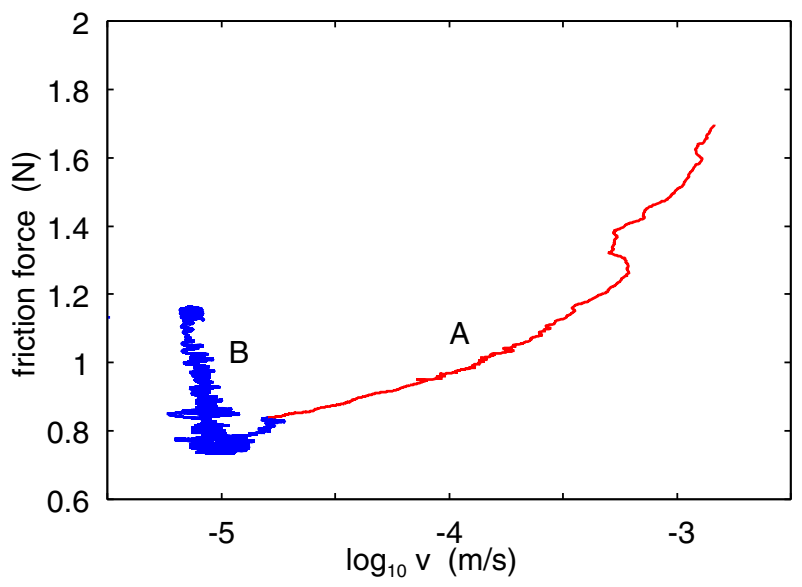

Fig. 29 The friction force as a function of the logarithm of the sliding velocity of the stopper with the $\mathrm{O}$-ring $\mathrm{O} 2$ with closed needle and $F_{x}=2 \mathrm{~N}$. The red part of the curve is the fast air compression part, and the blue part the following slow (air leakage) part of the friction curve
Funding Open Access funding enabled and organized by Projekt DEAL.

Open Access This article is licensed under a Creative Commons Attribution 4.0 International License, which permits use, sharing, adaptation, distribution and reproduction in any medium or format, as long as you give appropriate credit to the original author(s) and the source, provide a link to the Creative Commons licence, and indicate if changes were made. The images or other third party material in this article are included in the article's Creative Commons licence, unless indicated otherwise in a credit line to the material. If material is not included in the article's Creative Commons licence and your intended use is not permitted by statutory regulation or exceeds the permitted use, you will need to obtain permission directly from the copyright holder. To view a copy of this licence, visit http://creativecommons.org/licenses/by/4.0/.

\section{References}

1. Persson, B.N.J.: Sliding Friction: Physical Principles and Applications. Springer, Heidelberg (2000)

2. Gnecco, E., Meyer, E.: Elements of Friction Theory and Nanotribology. Cambridge University Press, Cambridge (2015)

3. Israelachvili, J.N.: Intermolecular and Surface Forces, 3rd edn. Academic, London (2011)

4. Barber, J.R.: Contact Mechanics, Solid Mechanics and Its Applications. Springer, London (2018)

5. Persson, B.N.J.: Contact mechanics for randomly rough surfaces. Surf. Sci. Rep. 61, 201 (2006)

6. Persson, B.N.J., Albohr, O., Tartaglino, U., Volokitin, A.I., Tosatti, E.: On the nature of surface roughness with application to contact mechanics, sealing, rubber friction and adhesion. J. Phys. Condens. Matter 17, R1 (2005)

7. Müser, M.H., Dapp, W.B., Bugnicourt, R., Sainsot, P., Lesaffre, N., Lubrecht, T.A., Persson, B.N.J., Harris, K., Bennett, A., Schulze, K., Rohde, S., Ifju, P., Sawyer, W.G., Angelini, T., Esfahani, H.A., Kadkhodaei, M., Akbarzadeh, S., Wu, J.-J., Vorlaufer, G., Vernes, A., Solhjoo, S., Vakis, A.I., Jackson, R.L., Xu, Y., Streator, J., Rostami, A., Dini, D., Medina, S., Carbone, G., Bottiglione, F., Afferrante, L., Monti, J., Pastewka, L., Robbins, M.O., Greenwood, J.A.: Meeting the contact-mechanics challenge. Tribol. Lett. 65, 118 (2017)

8. Vakis, A.I., Yastrebov, V.A., Scheibert, J., Minfray, C., Nicola, L., Dini, D., Almqvist, A., Paggi, M., Lee, S., Limbert, G., Molinari, J.F., Anciaux, G., Aghababaei, R., Restrepo, S.E., Papangelo, A., Cammarata, A., Nicolini, P., Putignano, C., Carbone, G., Ciavarella, M., Stupkiewicz, S., Lengiewicz, J., Costagliola, G., Bosia, F., Guarino, R., Pugno, N.M., Müser, M.H.: Modeling and simulation in tribology across scales: An overview. Tribol. Int. 125, 169-199 (2021)

9. Dapp, W.B., Lücke, A., Persson, B.N.J., Müser, M.H.: Self-affine elastic contacts: percolation and leakage. Phys. Rev. Lett. 108, 244301 (2012)

10. Persson, B.N.J., Yang, C.: Theory of the leak-rate of seals. J. Phys.: Condens. Matter 20, 315011 (2008)

11. Lorenz, B., Persson, B.N.J.: Leak rate of seals: effective-medium theory and comparison with experiment. Eur. Phys. J. E 31, 159 (2010)

12. Lorenz, B., Persson, B.N.J.: Leak rate of seals: comparison of theory with experiment/. EPL 86, 44006 (2009)

13. Bottiglione, F., Carbone, G., Mangialardi, L., Mantriota, G.: Leakage mechanism in flat seals. J. Appl. Phys. 106, 104902 (2009)

14. Persson, B.N.J.: Interfacial fluid flow for systems with anisotropic roughness. Eur. Phys. J. E 43, 1 (2020) 
15. Wang, A., Müser, M.H.: Percolation and Reynolds flow in elastic contacts of isotropic and anisotropic. Randomly rough surfaces. Tribol. Lett. 69, 1 (2021)

16. Persson, B.N.J.: Comments on the theory of fluid flow between solids with anisotropic roughness. Tribol. Lett. 69, 1 (2021)

17. Persson, B.N.J.: Theory of rubber friction and contact mechanics. J. Chem. Phys. 115, 3840 (2001)

18. Yang, C., Persson, B.N.J.: Contact mechanics: contact area and interfacial separation from small contact to full contact. J. Phys. Condens. Matter 20, 215214 (2008)

19. Afferrante, L., Bottiglione, F., Putignano, C., Persson, B.N.J., Carbone, G.: Elastic contact mechanics of randomly rough surfaces: an assessment of advanced asperity models and Persson's theory. Tribol. Lett. 66, 1 (2018)

20. Almqvist, A., Campana, C., Prodanov, N., Persson, B.N.J.: Interfacial separation between elastic solids with randomly rough surfaces: comparison between theory and numerical techniques. J. Mech. Phys. Solids 59, 2355 (2012)

21. Lorenz, B., Persson, B.N.J.: Time-dependent fluid squeeze-out between solids with rough surfaces. Eur. Phys. J. E 32, 281 (2010)

22. Beylich, A.E.: Solving the kinetic equation for all Knudsen numbers. Phys. Fluids 12, 444 (2000)

23. Jeans, J.: An Introduction to the Kinetic Theory of Gases, 1st edn. Cambridge University Press, Cambridge (2009)

24. Nacer, M.H.: Tangential Momentum Accomodation Coefficient in Microchannels with Different Surface Materials, PhD thesis, Marseille (2012)

25. Nacer, M.H., Graur, I., Perrier, P.: Mass flow measurement through rectangular microchannel from hydrodynamic to near free molecular regimes. La Houille Blanche 4, 49-54 (2011)
26. Pelaez, S.S., Mahler, H.C., Herdlitschka, C., Wertli, T., Kahl, M., Koulov, A., Matter, A., Singh, S.K., Widmer, M., Germershaus, O., Mathaes, R.: Comparing physical container closure integrity test methods and artificial leak methodologies. PDA J. Pharm. Sci. Technol. 73, 220 (2019)

27. Pelaez, S.S., Mahler, H.C., Matter, A., Koulov, A., Singh, S.K., Germershaus, O., Mathaes, R.: Container closure integrity testing of prefilled syringes. J. Pharm. Sci. 107, 2091 (2018)

28. Nieto, A., Roehl, H., Brown, H., Adler, M., Chalus, P., Mahler, H.C.: Artificial leaks in container closure integrity testing: nonlinear finite element simulation of aperture size originated by a copper wire sandwiched between the stopper and the glass vial. PDA J. Pharm. Sci. Technol. 70, 313 (2016)

29. Van Amerongen, G.J.: The permeability of different rubbers to gases and its relation to diffusivity and solubility. J. Appl. Phys. 17, 972 (1946)

30. Rutherford, S.W., Limmer, D.T., Smith, M.G., Honnell, K.G.: Gas transport in ethyleneepropyleneediene (EPDM) elastomer: molecular simulation and experimental study. Polymer 48, 6719 (2007)

31. Nieto, A., Roehl, H., Brown, H., Adler, M., Chalus, P., Mahler, H.-C.: Artificial leaks in container closure integrity testing: nonlinear finite element simulation of aperture size originated by a copper wire sandwiched between the stopper and the glass vial. PDA J. Pharm. Sci. Technol. 70, 313 (2016)

32. Arnold, J.C., Hutchings, I.M.: The erosive wear of elastomers. J. Nat. Rubber Res. 6, 241 (1991)

Publisher's Note Springer Nature remains neutral with regard to jurisdictional claims in published maps and institutional affiliations. 